\title{
Contribución al estudio de la resistencia química de los cementos. Comportamiento mecánico de un cemento portland resistente al yeso (2. ${ }^{\mathrm{a}}$ parte)
}

DEMETRIO GASPAR-TEBAR*, JOSE LUIS SAGRERA-MORENO* y VICTORIANO GONZALEZ-VILA**

\begin{abstract}
RES UMEN
En el presente trabajo, que completa el iniciado en (I), se estudia la evolución de las resistencias mecánicas a flexotraccion de diversas series de probetas de mortero (1:3) de $1 \times 1 \times 6 \mathrm{~cm}$ (12 probetas/serie/edad/ disolucion), hechas con un cemento portland industrial resistente al yeso (P-450-Y, segün $R C-75)$, sumergidas en agua potable filtrada y' en las siguientes disoluciones agresivas: saturada de sulfato de calcio, de $\mathrm{MgSO}_{4} .7 \mathrm{H}_{2} \mathrm{O}$ $(3,22 \mathrm{~g} / \mathrm{l})$, de $\mathrm{MgSO}_{4} .7 \mathrm{H}_{2} \mathrm{O}(32,2 \mathrm{~g} / \mathrm{l})$, saturada de sulfato de calcio más $\mathrm{MgSO}_{4} \cdot 7 \mathrm{H}_{2} \mathrm{O}(3,22 \mathrm{~g} / \mathrm{l})$, saturada de sulfato de calcio más $\mathrm{MgSO}_{4} .7 \mathrm{H}_{2} \mathrm{O}(32,2 \mathrm{~g} / \mathrm{l})$ y agua de mar artificial ASTM D 1141-75 durante 56- 90 $180-360-540-720-900$ y 1.080 dias, después del periodo de curado $(1+21$ dias $)$.
\end{abstract}

Así mismo, se estudia la variación de dichas resistencias mecánicas a flexotracción $y$ compresion de probetas de mortero $(1: 3)$ de $4 \times 4 \times 16 \mathrm{~cm}$, a tracción centrifuga de probetas de mortero de $7 \times 7 \times 28 \mathrm{~cm}$ y a

flexotracción y' compresión de probetas de hormigón de $10 \times 10 \times 40 \mathrm{~cm}$, hechas con el cemento mencionado $(P-450-Y)$, cuando se sumergen en agua potable !. en cinco disoluciones agresivas (disolución saturada de sulfato de calcio, disolución de $\mathrm{Na}_{2} \mathrm{SO}_{4}(2,1 \mathrm{~g} / \mathrm{l})$, disolución de $\mathrm{MgSO}_{4} .7 \mathrm{H}_{2} \mathrm{O}(3,22 \mathrm{~g} / \mathrm{l})$, disolución de $\mathrm{Na}_{2} \mathrm{SO}_{4}(3,5 \mathrm{~g} / \mathrm{l})+\mathrm{NaCl}(20,0 \mathrm{~g} / \mathrm{l})$ y agua de mar artificial ASTM D 1141-75] durante 6 - 12 - 18 - 24 $30-36-42-48-54$ y' 60 meses, después del periodo de curado $(1+30$ dias $)$.

Los resultados obtenidos han permitido establecer una graduación del cemento estudiado según su comportamiento, frente a las mencionadas disuluciones agresivas.

\author{
$S U M M A R Y$
}

This work, a completion of which started in (1), studies the evolution of the mechanical flexural strain of different ranges of mortar samples of $1 \times 1 \times 6 \mathrm{~cm}$ (12 samples/ranges/age/solution) made with sulphoresistant portland cement $(P-450-Y$, according to $R C$-75), submerged in filtered drinking water and in the following aggressive solutions: saturated of $\mathrm{CaSO}_{4} .2 \mathrm{H}_{2} \mathrm{O}$, saturated of $\mathrm{CaSO}_{4} .2 \mathrm{H}_{2} \mathrm{O}$, of $\mathrm{MgSO}_{4} .7 \mathrm{H}_{2} \mathrm{O}(3.22 \mathrm{~g} / \mathrm{l})$, of $\mathrm{MgSO}_{4} .7 \mathrm{H}_{2} \mathrm{O}(32.2 \mathrm{~g} / \mathrm{l})$, saturated of $\mathrm{CaSO}_{4} .2 \mathrm{H}_{2} \mathrm{O}$ plus $\mathrm{MgSO}_{4} .7 \mathrm{H}_{2} \mathrm{O}(3.22 \mathrm{~g} / \mathrm{l})$ saturated of $\mathrm{CaSO}_{4} .2 \mathrm{H}_{2} \mathrm{O}$ plus $\mathrm{MgSO}_{4} .7 \mathrm{H}_{2} \mathrm{O}(32.2 \mathrm{~g} / \mathrm{l})$ and artificial ASTM D 1141-75 sea water, during $56-90-180-360-540-720-900$ and 1.080 days, after the curing period $(1+21$ days $)$.

It also studies the variation of those mechanical flexural strain and compression strain of $4 \times 4 \times 16 \mathrm{~cm}$ mortar samples $(1: 3)$, to centrifugal tension of $7 \times 7 \times 28 \mathrm{~cm}$ mortar samples and to flexural strain and compression strain of $10 \times 10 \times 40 \mathrm{~cm}$ concrete samples made with the mentioned cement $(P-450-Y)$, when they are submerged in drinking water and in five agressive solutions $\left(\mathrm{CaSO}_{4} \cdot 2 \mathrm{H}_{2} \mathrm{O}\right.$ saturated solution, $\mathrm{Na}_{2} \mathrm{SO}_{4}(2.1 \mathrm{~g} / \mathrm{l})$ solution, $\mathrm{MgSO}_{4} .7 \mathrm{H}_{2} \mathrm{O}(3.22 \mathrm{~g} / \mathrm{l})$ solution, $\mathrm{Na}_{2} \mathrm{SO}_{4}(3.5 \mathrm{~g} / \mathrm{l})$ plus $\mathrm{NaCl}(20.0 \mathrm{~g} / \mathrm{l})$ solution and artificial ASTM D 1141-75 sea water during 6- 12 $18-24-30-36-42-48-54$ and 60 months, after the curing period $(1+30$ days $)$.

The results obtained allow to stablish a range of the studied cement according to its behaviour, against the mentioned agressive solutions.

\section{INTRODUCCION}

En un trabajo anterior (1) se dio cuenta del comportamiento quimico-resistente de un cemento portland industrial (P-450-Y) de fabricación española, análogo al estudiado en otros trabajos (2) (3) (4) (5) (6) (7), frente al sulfato de calcio dihidratado por los métodos de Le Chatelier-

* IETcc/CSIC.

** Cementos del Atlántico, S. A. 
Anstett y por el descrito en la norma ASTM C 452-73, asi como frente a las disoluciones agresivas que se incluyen en el apartado 2 de este artículo, por los métodos de Wittekindt y de Koch-Steinegger.

En otros trabajos (2) (3) (4) (5) (6) (7) se estudió:

a) la evolución de las resistencias mecánicas a flexotracción de las probetas de mortero $(1: 3)$ de $1 \times 1 \times 6 \mathrm{~cm}$, hechas con el cemento portland industrial resistente al yeso, objeto del presente artículo, sumergidas en agua potable filtrada (2), unas series de 12 probetas, en agua de mar artificial (2), otras series análogas, y en una disolución saturada de yeso (3), durante $56-90-180$ y 360 dias, después del periodo de curado $(1+21$ días $)$,

b) la composición estructural de las nuevas fases sólidas formadas en los medios de curado y de conservación (agua potable filtrada) (4), de conservación-ataque (agua de mar artificial) (5),

c) las características estructurales de la fracción enriquecida (cemento hidratado y cemento hidratado-atacado) extraída de uno de los prismas de mortero de cada serie de probetas sumergidas en agua potable filtrada (4) y en agua de mar artificial (5),

d) la variación de la concentración de los iones calcio (II) y sulfato (II) en los medios de curado y de conservación (agua potable filtrada) (6) y de conservación-ataque (agua de mar artificial) (7) en donde han estado sumergidas las diversas series de probetas de mortero durante los períodos de tiempo mencionados anteriormente, así como de las fases sólidas formadas, y la evolución del $\mathrm{pH}$ de dichos medios. Además de la concentración de los iones señalados, en el agua de mar artificial se ha determinado, en cada caso, el contenido de los iones magnesio (II) y cloruro (I).

\section{PARTE EXPERIMENTAL}

En este trabajo se estudia el comportamiento quimico-resistente del cemento portland industrial resistente al yeso, de fabricación española, que se utilizó en (1), cuando las probetas de mortero y de hormigón, hechas con dicho cemento, se someten a la acción de las siguientes disoluciones agresivas:

Disolución 1.-Disolución saturada de sulfato de calcio $\left(\sim 2,1 \mathrm{~g} / l\right.$ de $\left.\mathrm{CaSO}_{4} \cdot 2 \mathrm{H}_{2} \mathrm{O}\right)$.

Disolución 2.-Disolución de $\mathrm{Na}_{2} \mathrm{SO}_{4}(2,1 \mathrm{~g} / l)$.

Disolución 3.-Disolución de $\mathrm{MgSO}_{4} \cdot 7 \mathrm{H}_{2} \mathrm{O}(3,22 \mathrm{~g} / l)$.

Disolución 4.-Disolución de $\mathrm{MgSO}_{4} \cdot 7 \mathrm{H}_{2} \mathrm{O}(32,2 \mathrm{~g} / \mathrm{l})$.

Disolución 5.-Disolución saturada de sulfato de calcio $+\mathrm{MgSO}_{4} \cdot 7 \mathrm{H}_{2} \mathrm{O}(3,22 \mathrm{~g} / l)$.

Disolución 6.-Disolución saturada de sulfato de calcio $+\mathrm{MgSO}_{4} \cdot 7 \mathrm{H}_{2} \mathrm{O}(32,2 \mathrm{~g} / l)$.

Disolución 7.-Disolución de $\mathrm{Na}_{2} \mathrm{SO}_{4}(3,5 \mathrm{~g} / l)+\mathrm{NaCl}(20,0 \mathrm{~g} / l)$.

Disolución 8.-Agua de mar artificial ASTM D 1141-75,

$\mathrm{y}$, además, agua potable o agua potable filtrada, que se han designado disolución 0 , determinando la evolución de las resistencias mecánicas a:

$1^{\circ}$ ) flexotracción de las probetas de mortero $(1: 3)$ de $1 \times 1 \times 6 \mathrm{~cm}$, sumergidas en las disoluciones agresivas $1,3,4,5,6,8 \mathrm{y}$, además, en agua potable filtrada (que se ha denominado disolución 0$)$, después del periodo de curado $(1+21$ dias) durante 56,90 , $180,360,540,720,900$ y 1.080 dias, 
$\left.2 .^{\circ}\right)$ tracción centrífuga de las probetas de mortero $(1: 3)$ de $7 \times 7 \times 28 \mathrm{~cm}$, sumergidas en agua potable (disolución 0 ) y en las disoluciones agresivas $1,2,3,7,8$, después del período de curado $(1+30$ dias $)$, durante $1 / 2,1,11 / 2,2,21 / 2,3,31 / 2,4,41 / 2$ y 5 años. Se ha utilizado la máquina de rotura de Berthier,

$\left.3{ }^{\circ}\right)$ flexotracción y compresión de probetas de mortero $(1: 3)$ de $4 \times 4 \times 16 \mathrm{~cm}$, sumergidas en agua potable (designada disolución 0 ) y en las disoluciones agresivas señaladas en el punto $b$, después del periodo de curado $(1+30$ dias $)$, durante los mismos periodos de tiempo, que se han denominado periodos de conservación-ataque,

$\left.4^{\circ}{ }^{\circ}\right)$ flexotracción y compresión de probetas de hormigón de $10 \times 10 \times 40 \mathrm{~cm}$ sumergidas en agua potable y en las disoluciones agresivas indicadas en el punto $b$, después del período de curado $(1 \times 30$ días), durante los mismos períodos de tiempo de conservación-ataque.

\subsection{Materiales utilizados}

\section{a) Cemento}

Se ha utilizado el cemento portland industrial resistente al yeso (P-450-Y) de fabricación española, que se estudió y utilizó en (1), el cual tiene un contenido calculado (Bogue) nulo de $\mathrm{C}_{3} \mathrm{~A}$, del 10,8 \% de $\mathrm{C}_{4} \mathrm{AF}$ y del $81,7 \%$ de $\mathrm{C}_{3} \mathrm{~S}(75,6 \%)+\mathrm{C}_{2} \mathrm{~S}(6,1 \%)$.

b) Arena utilizada para preparar las probetas de mortero de $1 \times 1 \times 6 \mathrm{~cm}$

Se ha usado la arena natural (El Espirdo-Segovia) análoga a la empleada para preparar las probetas de morteros normalizados (RC-75), con un contenido de $\mathrm{SiO}_{2}$ superior a $99 \%$. El tamaño de grano está comprendido entre 1 y $1,5 \mathrm{~mm}$.

c) Arena utilizada para preparar las probetas de mortero de $4 \times 4 \times 16 \mathrm{~cm} y$ de $7 \times 7 \times 28 \mathrm{~cm}$

Se ha empleado la arena especificada en el Pliego General de Condiciones para la Recepción de Cementos (RC-75).

d) Aridos utilizados para preparar las probetas de hormigón de $10 \times 10 \times$ $\times 40 \mathrm{~cm}$

Para fabricar las probetas de hormigón de $10 \times 10 \times 40 \mathrm{~cm}$ se han utilizado áridos rodados (silícicos) lavados, que responden a la curva granulométrica de Bolomey.

\subsection{Disoluciones agresivas}

Se han utilizado las disoluciones agresivas que figuran en la tabla 1, las cuales se han preparado como se señala en (1). Los iones determinados y su concentración se incluyen en dicha tabla 1 .
T ABLA 1

Concentración $(g / l)$ de los iones $\mathrm{SO}_{4}(\mathrm{II})$, $\mathrm{Cl}$ (I), Ca (II) y $\mathrm{Mg}$ (II) de las disoluciones agresivas y del agua potable

\begin{tabular}{|c|c|c|c|c|}
\hline \multirow{2}{*}{$\begin{array}{c}\text { Disolución } \\
\text { n.o }\end{array}$} & \multicolumn{4}{|c|}{ Concentración, g/l } \\
\cline { 2 - 5 } & $\mathbf{S O}_{\mathbf{4}}$ (II) & Cl (I) & Ca (II) & Mg (II) \\
\hline 1 & 1,010 & - & 0,420 & - \\
2 & 1,210 & - & - & - \\
3 & 1,263 & - & - & 0,318 \\
4 & 12,630 & - & - & 3,180 \\
5 & 2,273 & - & 0,420 & 0,318 \\
6 & 13,280 & - & 0.420 & 3,180 \\
7 & 2,494 & 12,132 & - & - \\
8 & 2,928 & 19,585 & 0.521 & 1,347 \\
0 (agua & 0,003 & 0,007 & 0,005 & $0.001_{6}$ \\
potable & & & & \\
o agua \\
potable \\
filtrada)
\end{tabular}




\subsection{Preparación, curado y conservación de las probetas de mortero y de hormigón}

a) Probetas de mortero de $1 \times 1 \times 6 \mathrm{~cm}$

Las probetas de mortero de $1 \times 1 \times 6 \mathrm{~cm}$ se han preparado, curado y conservado según se señala en (8). Dichas probetas se han curado 1 día en cámara húmeda a $20 \pm 1^{\circ} \mathrm{C}$, con una humedad relativa superior al $95 \%$, y a continuación se han sumergido en agua potable filtrada durante 21 días; posteriormente, se han sumergido, una serie de 12 probetas, en $800 \mathrm{ml}$ de las disoluciones $1,3,4,5,6,8$ y agua potable filtrada (disolución 0 ) en donde han permanecido hasta el momento de su rotura.

Para realizar este estudio, se han preparado 56 series de probetas (12 probetas/serie), una por edad y por disolución, las cuales se han curado y conservado en los recipientes correspondientes, que se han situado en un recinto del laboratorio.

\section{b) Probetas de mortero de $4 \times 4 \times 16 \mathrm{~cm}$}

Las distintas series de probetas de mortero de $4 \times 4 \times 16 \mathrm{~cm}(10$ probetas por serie, por edad $\mathrm{y}$ por disolución) se han preparado de acuerdo con el procedimiento operatorio descrito en el Pliego General de Condiciones para la Recepción de Cementos (RC-75).

Las diversas series de probetas de mortero se han curado en una cámara húmeda con una humedad relativa superior al $95 \%$, a $20 \pm 2^{\circ} \mathrm{C}$, durante las primeras 24 horas y durante 30 días bajo agua potable a $20 \pm 2^{\circ} \mathrm{C}$. A continuación, se han sumergido hasta el momento de su rotura en unos recipientes de asbesto-cemento protegidos con un revestimiento a base de resina epoxi, que contienen las disoluciones agresivas 1, 2, 3, 7, 8 y agua potable (disolución 0). Dichos depósitos se han situado en el recinto del Instituto, sometidos a las condiciones climáticas ambientes.

Las probetas se han colocado en posición vertical, separadas entre sí por una capa de disolución de unos $5 \mathrm{~cm}$.

Las disoluciones agresivas se han renovado cuando la concentración de iones sulfato en dichas disoluciones era inferior a $500 \mathrm{mg} / \mathrm{l}$.

\section{c) Probetas de mortero de $7 \times 7 \times 28 \mathrm{~cm}$}

Las distintas series de probetas de mortero de $7 \times 7 \times 28 \mathrm{~cm}(10$ probetas por serie, por edad y por disolución) se han preparado, curado y conservado, siguiendo unos procedimientos análogos a los señalados en el apartado anterior (b), utilizando las mismas dosificaciones, las mismas condiciones y periodos de tiempo de curado y conservación; asi mismo, las probetas se han colocado en posición vertical.

Las disoluciones agresivas se han renovado cuando la concentración de iones sulfato en las mencionadas disoluciones era inferior a $500 \mathrm{mg} / \mathrm{l}$.

\section{d) Probetas de hormigón de $10 \times 10 \times 40 \mathrm{~cm}$}

Las series de probetas de hormigón de $10 \times 10 \times 40 \mathrm{~cm}(10$ probetas por serie, por edad y por disolución) se han preparado ccn áridos rodados silícicos, lavados, respondiendo a la curva granulométrica de Bolomey. Se han empleado $400 \mathrm{~kg}$ de cemento por $\mathrm{m}^{3}$ de hormigón y una relación agua/cemento $=0,45$. 
Cada 4 probetas se han vibrado, en una mesa vibradora, a $2.800 \mathrm{rpm}$ durante dos minutos (1 minuto por tongada/dos tongadas probeta).

Las diversas series de probetas de hormigón se han conservado las primeras 24 horas en un laboratorio, situado en el recinto del Instituto, y a continuación bajo agua potable durante 30 dias en unas balsas situadas al aire libre en el mencionado recinto del Instituto (figura 1). Posteriormente, se han sumergido en las disoluciones agresivas $1,2,3,7,8$ (tabla 1) y en agua potable (disolución 0), que se encuentran en unas balsas análogas a las anteriores. Dichas balsas están forradas con un mortero de cemento $\mathrm{y}$ protegidas por un revestimiento a base de una resina epoxi. Cada balsa contiene $2.000 l$ de cada una de las diversas disoluciones agresivas y otra balsa $2.000 l$ de agua potable.

Las probetas de hormigón se han colocado en posición vertical, como en los casos b y c.

Lo mismo que en los casos b y c anteriores, las disoluciores agresivas se han renovado cuando la concentración de iones sulfato era inferior a $500 \mathrm{mg} / l$.



Fig. 1.-Vista de los depósitos de conservación de las probetas de hormigón.

\section{RESULTADOS OBTENIDOS}

\subsection{Evolución de las resistencias mecánicas, a flexotracción, de las probetas de mortero de $1 \times 1 \times 6 \mathrm{~cm}$}

En la figura 2 se ha representado, en función del tiempo de conservación, la evolución de las resistencias mecánicas a flexotracción de las series de probetas de mortero $(1: 3)$ de $1 \times 1 \times 6 \mathrm{~cm}$ - valores medios de 12 probetas por serie, por agresivo y por edad-, sumergidas después del periodo de curado, durante 56 días, $3-6-12-18-24-30$ y 36 meses, en las disoluciones agresivas $1,3,4,5,6,8$ (tabla 1 ) y en agua potable filtrada, que se ha designado disolución 0 .

En dicha figura se aprecia que las mencionadas resistencias mecánicas, con relación a las alcanzadas por las series de probetas conservadas en agua potable filtrada, es:

a) mayor a todas las edades, para el caso de las series de probetas sumergidas en la disolución saturada de yeso con $32,2 \mathrm{~g} / l$ de $\mathrm{MgSO}_{4} .7 \mathrm{H}_{2} \mathrm{O}$ y en la disolución de $\mathrm{MgSO}_{4} .7 \mathrm{H}_{2} \mathrm{O}$ $(32,2 \mathrm{~g} / l)$; en el caso de las probetas conservadas en la disolución saturada de yeso - por regla general- es mayor, excepto para dos edades que es del mismo orden,

b) del mismo orden, o con variaciones en más o en menos para determinadas edades, para los casos de las series de probetas sumergidas en la disolución de $\mathrm{MgSO}_{4} \cdot 7 \mathrm{H}_{2} \mathrm{O}(3,22 \mathrm{~g} / l)$ y de la disolución saturada de yeso más $3,22 \mathrm{~g} / l$ de $\mathrm{MgSO}_{4} \cdot 7 \mathrm{H}_{2} \mathrm{O}$,

c) menor, con tendencia a igualarse, para el caso de las series de probetas conservadas en agua de mar artificial ASTM D 1141-75.

Los valores obtenidos en las distintas series de probetas de mortero, sumergidas en las mencionadas disoluciones agresivas, permiten establecer, de mayor a menor, la siguiente graduación: 
Disolución saturada de yeso más $32,2 \mathrm{~g} / l$ de $\mathrm{MgSO}_{4} \cdot 7 \mathrm{H}_{2} \mathrm{O}>$ Disolución de $\mathrm{MgSO}_{4} \cdot 7 \mathrm{H}_{2} \mathrm{O}$ $(32,2 \mathrm{~g} / l)>$ Disolución saturada de yeso $>$ Agua potable filtrada $\simeq$ Disolución de $\mathrm{MgSO}_{4} \cdot 7 \mathrm{H}_{2} \mathrm{O}$ $(3,22 \mathrm{~g} / l) \simeq$ Disolución saturada de yeso más $3,22 \mathrm{~g} / l$ de $\mathrm{MgSO}_{4} \cdot 7 \mathrm{H}_{2} \mathrm{O}>$ Agua de mar artificial ASTM D 1141-75.

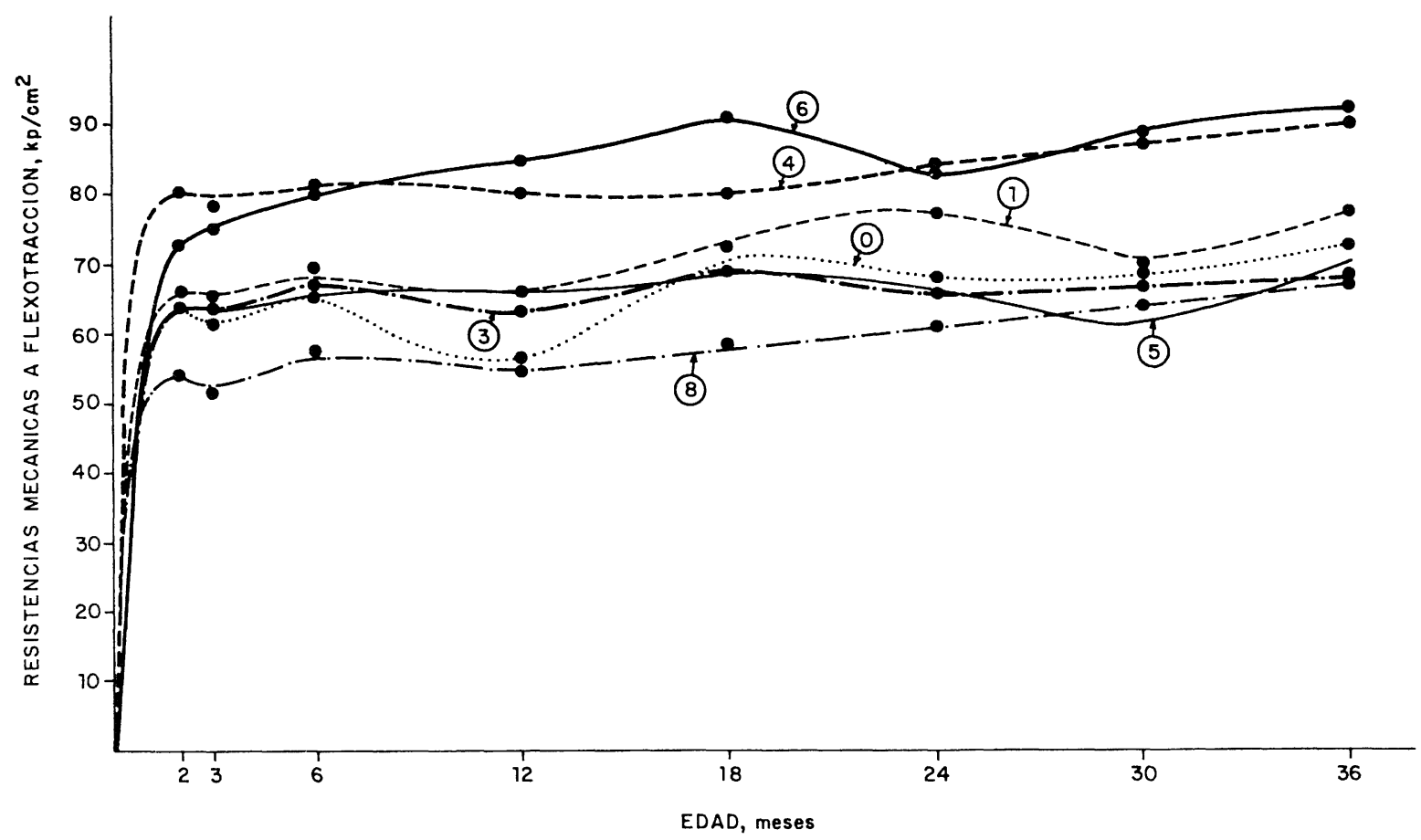

Fig. 2.-Evolución de las resistencias mecánicas, a flexotracción, de las probetas de mortero $1 \times 1 \times 6 \mathrm{~cm}$, sumergidas en:

1. disolución saturada de $\mathrm{CaSO}_{4} \cdot 2 \mathrm{H}_{2} \mathrm{O}$,

3. disolución de $\mathrm{MgSO}_{4} .7 \mathrm{H}_{2} \mathrm{O}(3,22 \mathrm{~g} / l)$,

4. disolución de $\mathrm{MgSO}_{4} \cdot 7 \mathrm{H}_{2} \mathrm{O}(32,2 \mathrm{~g} / l)$,

5. disolución saturada de $\mathrm{CaSO}_{4} \cdot 2 \mathrm{H}_{2} \mathrm{O}+\mathrm{MgSO}_{4} \cdot 7 \mathrm{H}_{2} \mathrm{O}(3,22 \mathrm{~g} / l)$,

6. disolución saturada de $\mathrm{CaSO}_{4} \cdot 2 \mathrm{H}_{2} \mathrm{O}+\mathrm{MgSO}_{4} \cdot 7 \mathrm{H}_{2} \mathrm{O}(32,2 \mathrm{~g} / l)$,

8. agua de mar artificial ASTM D 1141-75,

0 . agua potable filtrada.

\subsection{Evolución de las resistencias mecánicas, a flexotracción y compresión, de las probetas de mortero de $4 \times 4 \times 16 \mathrm{~cm}$}

En las figuras 3 y 5 se han representado las resistencias mecánicas, a flexotracción y compresión, de las probetas de mortero de $4 \times 4 \times 16 \mathrm{~cm}$ (RC-75) sumergidas en las disoluciones agresivas 1, 2, 3, 7, 8 y agua potable (que se ha designado disolución 0) (tabla 1), después del periodo de curado $(1+30$ días $)$, en función del tiempo de conservación $(6,12,18$, $24,30,36,42,48,54$ y 60 meses). En dichas figuras, para cada disolución y para cada edad, se ha representado el valor medio de los valores correspondientes a 10 probetas, en la primera figura, y a las 20 mitades de dichas probetas, en la segunda, asi como. los valores máximos y minimos obtenidos en cada serie. En la última edad, 60 meses, se han roto 6 probetas a flexotracción y las 12 mitades a compresión.

En la figura 3 se puede apreciar que las resistencias mecánicas a flexotracción de las series de probetas sumergidas en las disoluciones saturadas de yeso, de $\mathrm{MgSO}_{4} .7 \mathrm{H}_{2} \mathrm{O}(3,22 \mathrm{~g} / l)$, de $\mathrm{Na}_{2} \mathrm{SO}_{4}(2,1 \mathrm{~g} / l)$ y de $\mathrm{Na}_{2} \mathrm{SO}_{4}(3,5 \mathrm{~g} / l)$ más $\mathrm{NaCl}(20,0 \mathrm{~g} / l)$ son superiores que las correspondientes a las series de probetas conservadas bajo agua potable y éstas, a su vez, mayores que las de las series sumergidas en agua de mar artificial ASTM D 1141-75. La evolución de las resistencias, a lo largo de los cinco años, de las probetas de mortero sumergidas 
en las cuatro primeras disoluciones sigue un hábito análogo entre sí y al de las probetas sumergidas en agua potable. Las resistencias de las mencionadas series de probetas alcanzan, a los 6 meses, unos valores medios comprendidos entre 92,6 y $96,7 \mathrm{kp} / \mathrm{cm}^{2}$ y a los 60 meses entre 99 y $101,6 \mathrm{kp} / \mathrm{cm}^{2}$; valores que se obtienen a los 12 meses en la serie de probetas sumergidas en la disolución de $\mathrm{MgSO}_{4} .7 \mathrm{H}_{2} \mathrm{O}$, a los 18 meses en la disolución saturada de yeso, a los 30 - 36 meses en las disoluciones de $\mathrm{Na}_{2} \mathrm{SO}_{4}$ y de $\mathrm{Na}_{2} \mathrm{SO}_{4}+\mathrm{NaCl}$.

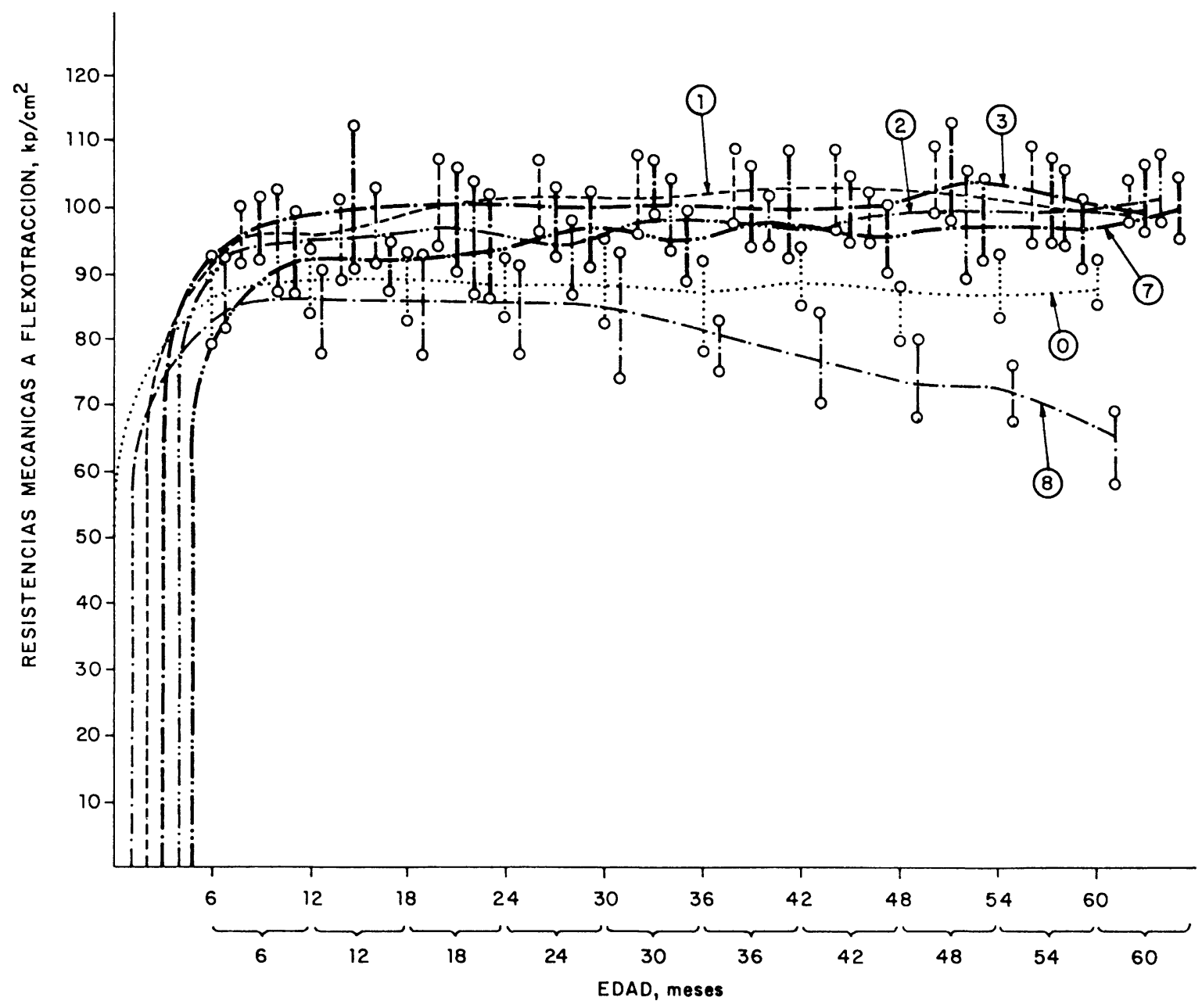

Fig. 3.-Evolución de las resistencias mecánicas, a flexotracción, de las probetas de mortero de $4 \times 4 \times 16 \mathrm{~cm}$, sumergidas en:

1. disolución saturada de $\mathrm{CaSO}_{4} \cdot 2 \mathrm{H}_{2} \mathrm{O}$,

2. disolución de $\mathrm{Na}_{2} \mathrm{SO}_{4}(2,1 \mathrm{~g} / l)$,

3. disolución de $\mathrm{MgSO}_{4} .7 \mathrm{H}_{2} \mathrm{O}(3,22 \mathrm{~g} / l)$,

7. disolución de $\mathrm{Na}_{2} \mathrm{SO}_{4}(3,5 \mathrm{~g} / l)+\mathrm{NaCl}(20,0 \mathrm{~g} / l)$,

8. agua de mar artificial ASTM D 1141-75,

0 . agua potable.

Las series de probetas conservadas en agua potable alcanzan, a los 6 meses, el valor medio $86,4 \mathrm{kp} / \mathrm{cm}^{2}$ y a los 60 meses, $88,4 \mathrm{kp} / \mathrm{cm}^{2}$; valor obtenido a los 24 meses.

Los valores alcanzados, a los 6 meses, por las probetas sumergidas en agua de mar artificial ASTM D 1141-75 $\left(86,5 \mathrm{kp} / \mathrm{cm}^{2}\right)$ permanecen, prácticamente, constantes hasta los 30 meses, disminuyendo a continuación.

En la figura 4 se han representado los valores correspondientes a la relación entre las 
resistencias mecánicas a flexotracción, para cada edad, de las diversas series de probetas sumergidas en las disoluciones agresivas $\left(R_{t}\right)$ y en agua potable $\left(R^{\prime}\right)$, en función del tiempo.

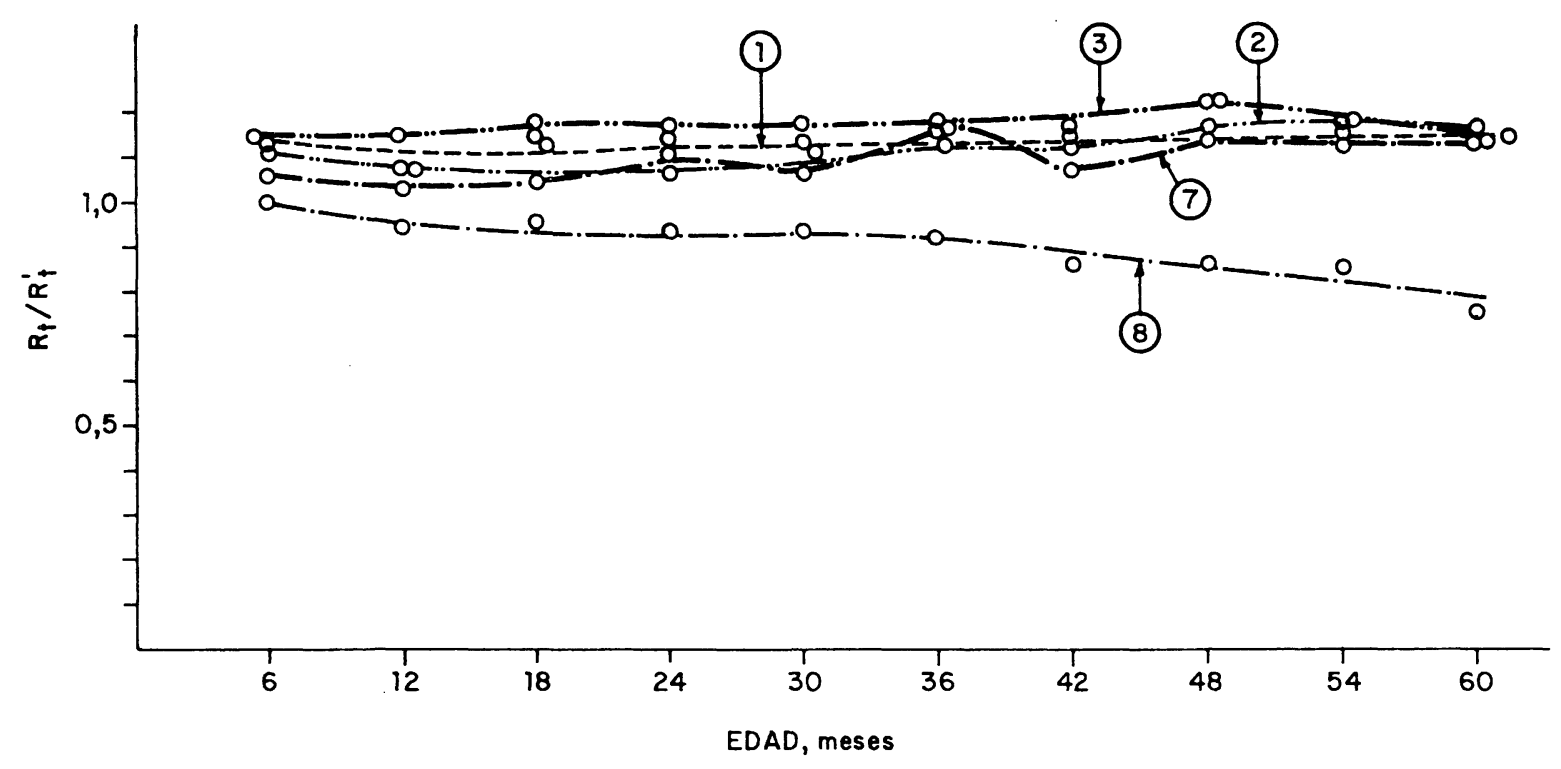

Fig. 4.--Evolución de los coeficientes $R_{\mathrm{t}} / R_{\mathrm{t}}$.

$R_{\mathrm{L}}=$ Resistencias mecánicas, a flexotracción, (disolución agresiva),

$R_{\mathrm{t}}^{\prime}=$ Resistencias mecánicas, a flexotracción, (agua potable),

1. disolución saturada de $\mathrm{CaSO}_{4} \cdot 2 \mathrm{H}_{2} \mathrm{O}$,

2. disolución de $\mathrm{Na}_{2} \mathrm{SO}_{4}(2,1 \mathrm{~g} / l)$,

3. disolución de $\mathrm{MgSO}_{4} .7 \mathrm{H}_{2} \mathrm{O}(3,22 \mathrm{~g} / l)$,

7. disolución de $\mathrm{Na}_{2} \mathrm{SO}_{4}(3,5 \mathrm{~g} / l)+\mathrm{NaCl}(20,0 \mathrm{~g} / l)$,

8. agua de mar artificial ASTM D 1141-75.

En dicha figura, se puede apreciar que los valores de dichos coeficientes son mayores que la unidad para los casos de las probetas sumergidas en las diversas disoluciones agresivas, excepto para el agua de mar artificial ASTM que son menores; en los primeros casos se encuentran comprendidos entre 1,07 y 1,23 y entre 0,72 y 1,00 para el último.

Las resistencias mecánicas, a compresión, en función del tiempo, de las probetas de mortero sumergidas en las disoluciones agresivas, con relación a las de las series de probetas conservadas en agua potable (figura 5) es del mismo orden que las correspondientes a las de las probetas sumergidas en la disolución de $\mathrm{Na}_{2} \mathrm{SO}_{4}+\mathrm{NaCl}$, menores que las de las series conservadas en las disoluciones de $\mathrm{Na}_{2} \mathrm{SO}_{4}$, saturada de yeso y de $\mathrm{MgSO}_{4} .7 \mathrm{H}_{2} \mathrm{O}$ y mayores que las correspondientes a las probetas sumergidas en agua de mar artificial ASTM D 1141-75.

La disolución saturada de sulfato de calcio se ha renovado a los siguientes periodos de tiempo: 4, 10, 18, 28 y 34 meses, la de $\mathrm{Na}_{2} \mathrm{SO}_{4}$, a los 18 meses y la de $\mathrm{MgSO}_{4} .7 \mathrm{H}_{2} \mathrm{O}$, a los 4 meses, por ser la concentración de iones sulfato inferior a $500 \mathrm{mg} / \mathrm{l}$.

El aspecto de las probetas sometidas a la acción de las diversas disoluciones, para todas las edades, era normal, no habiéndose observado alteraciones.

\subsection{Evolución de las resistencias mecánicas, a tracción centrifuga, de las probetas de mortero de $7 \times 7 \times 28 \mathrm{~cm}$}

La evolución de las resistencias mecánicas, a tracción centrifuga, de las probetas de mortero de $7 \times 7 \times 28 \mathrm{~cm}$, sumergidas en las disoluciones agresivas $1,2,3,7,8$ y agua potable (que se 


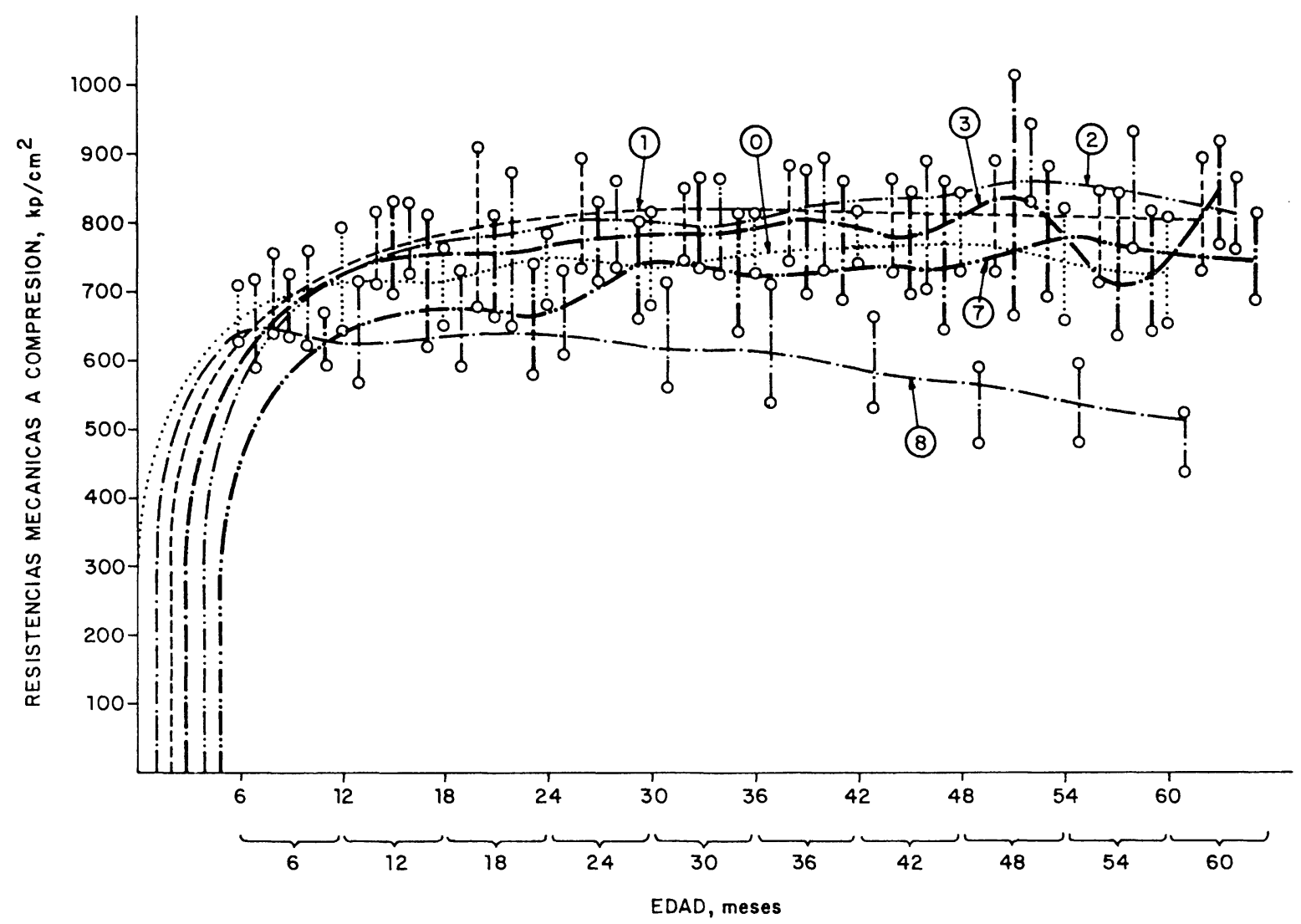

Fig. 5.-Evolución de las resistencias mecánicas, a compresión, de las probetas de mortero de $4 \times 4 \times 16 \mathrm{~cm}$, sumergidas en:

1. disolución saturada de $\mathrm{CaSO}_{4} \cdot 2 \mathrm{H}_{2} \mathrm{O}$,

2. disolución de $\mathrm{Na}_{2} \mathrm{SO}_{4}(2,1 \mathrm{~g} / l)$,

3. disolución de $\mathrm{MgSO}_{4} .7 \mathrm{H}_{2} \mathrm{O}(3,22 \mathrm{~g} / l)$,

7. disolución de $\mathrm{Na}_{2} \mathrm{SO}_{4}(3,5 \mathrm{~g} / 21)+\mathrm{NaCl}(20,0 \mathrm{~g} / l)$,

8. agua de mar artificial ASTM D 1141-75,

0 . agua potable.

ha designado disolución 0 ) (tabla 1), después del periodo de curado $(1+30$ dias) en función del tiempo de conservación $(6,12,18,36,42,48,54$ y 60 meses) se ha representado en la figura 6. Por avería de la máquina de ensayo de rotura de Berthier, no se han podido determinar las resistencias a las edades correspondientes a 24 y 30 meses.

La evolución de las mencionadas resistencias, a lo largo del tiempo, de las distintas series de probetas de mortero sigue un hábito análogo al correspondiente a las probetas conservadas en agua potable, formando un haz cuyos valores extremos se encuentran comprendidos entre $30,2 \mathrm{kp} / \mathrm{cm}^{2}$ y $38,6 \mathrm{kp} / \mathrm{cm}^{2}$. Los valores inferiores y superiores, más frecuentes para las distintas edades, corresponden a las series de probetas sumergidas en el agua de mar artificial ASTM D 1141-75 y en la disolución de $\mathrm{Na}_{2} \mathrm{SO}_{4}$, respectivamente.

Las disoluciones saturadas de sulfato de calcio, de $\mathrm{MgSO}_{4} \cdot 7 \mathrm{H}_{2} \mathrm{O}$ y la de agua de mar artificial ASTM D 1141-75 se han renovado a los periodos de tiempo: 6, 18, 26, 36 y 46 meses, la primera, 6 y 16 meses la segunda y 46 meses, la tercera, por ser la concentración de iones sulfato inferior a $500 \mathrm{mg} / l$.

El aspecto de las probetas, sometidas a la acción de las disoluciones, era normal a todas las edades, como se puede apreciar en la fig. 7 en donde, a titulo de ejemplo, se encuentra una fotografia de una de las probetas de las distintas series sometidas durante 5 años a la acción del agua potable y de las mencionadas diversas disoluciones agresivas. Las probetas sometidas a la acción del agua de mar aparecen recubiertas, parcialmente, por una capa de $\mathrm{Mg}(\mathrm{OH})_{2}$. 


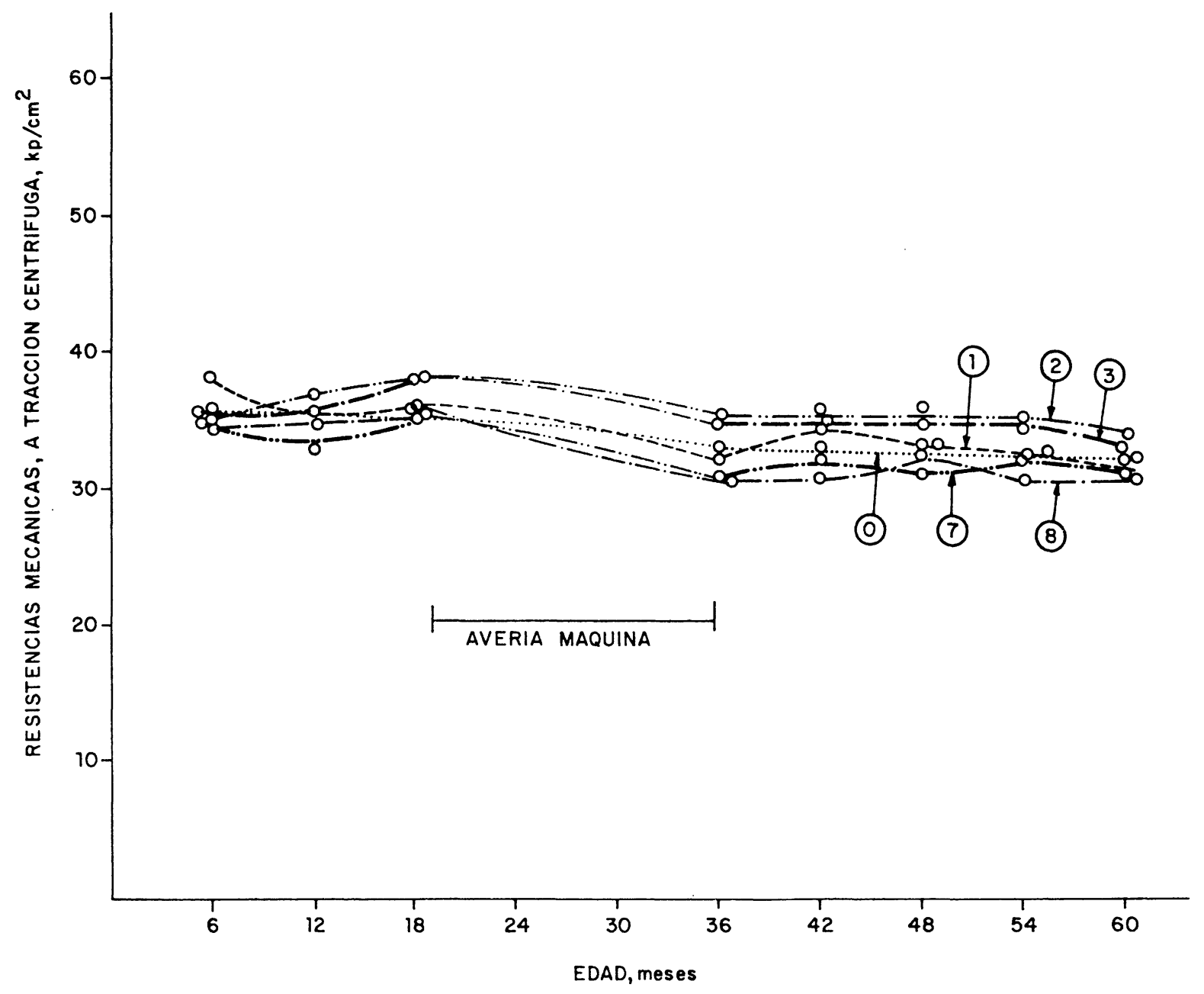

Fig. 6.-Evolución de las resistencias mecánicas, a tracción centrufuga, de probetas de mortero de $7 \times 7 \times 28 \mathrm{~cm}$, sumergidas en:

1. disolución saturada de $\mathrm{CaSO}_{4} \cdot 2 \mathrm{H}_{2} \mathrm{O}$,

2. disolución de $\mathrm{Na}_{2} \mathrm{SO}_{4}(2,1 \mathrm{~g} / l)$,

3. disolución de $\mathrm{MgSO}_{4} \cdot 7 \mathrm{H}_{2} \mathrm{O}(3,22 \mathrm{~g} / l)$,

7. disolución de $\mathrm{Na}_{2} \mathrm{SO}_{4}(3,5 \mathrm{~g} / l)+\mathrm{NaCl}(20,0 \mathrm{~g} / l)$,

8. agua de mar artificial ASTM D 1141-75,

0 . agua potable.

\subsection{Evolución de las resistencias mecánicas, a flexotracción y compresión, de las probetas de hormigón de $10 \times 10 \times 40 \mathrm{~cm}$}

En las figuras 8 y 9 se han representado las resistencias mecánicas, a flexotracción y compresión, de las probetas de hormigón de $10 \times 10 \times 40 \mathrm{~cm}$ sumergidas en las disoluciones agresivas $1,2,3,7,8$ y en agua potable (que se ha denominado disolución 0 ) (tabla 1), después del período de curado $(1+30$ dias), en funciọ́n del tiempo de conservación $(6,12,18,24,30$, $36,42,48,54$ y 60 meses). En dichas figuras se han representado, para cada disolución y para cada edad, los valores medios correspondientes a los valores individuales de 10 probetas (una serie), en la primera figura, y de 20 mitades de dichas probetas, en la segunda figura, así como los valores máximos y mínimos obtenidos en cada serie. En la última edad se han roto 6 probetas a flexotracción y sus 12 mitades a compresión.

En la figura 8 se puede apreciar que las resistencias mecánicas a flexotracción de las distintas series de probetas sumergidas en las diversas disoluciones agresivas son, por regla general, inferiores a las correspondientes a las de las probetas conservadas bajo agua potable y todas ellas mayores que las de las sumergidas en agua de mar artificial. 


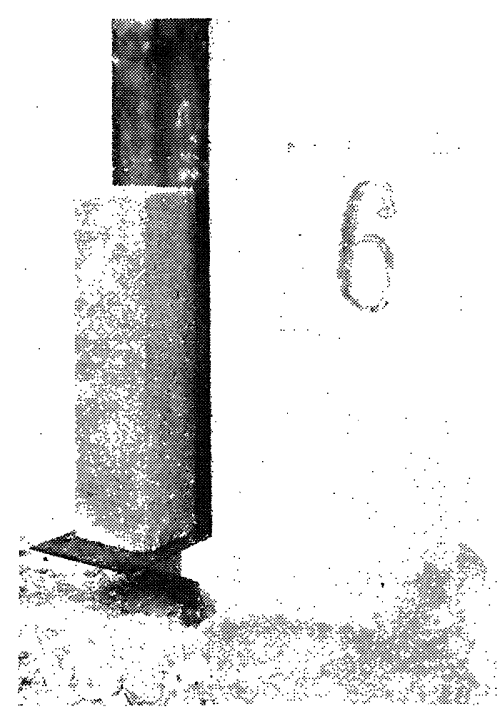

$\mathrm{CaSO}_{4} \cdot 2 \mathrm{H}_{2} \mathrm{O}:$ sat.

(60 meses)

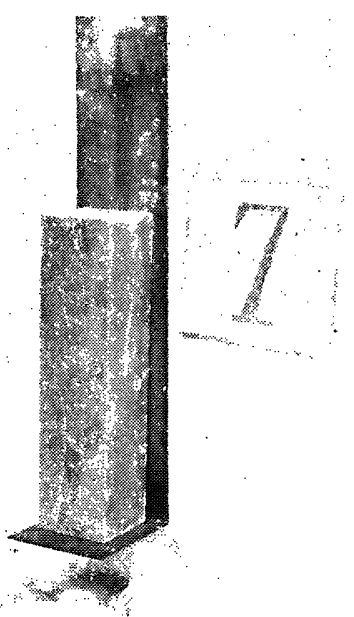

$\mathrm{Na}_{2} \mathrm{SO}_{4}+\mathrm{NaCl}$ (60 meses)

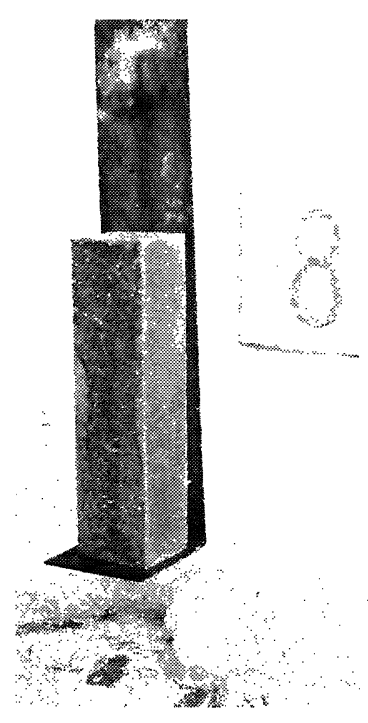

$\mathrm{Na}_{2} \mathrm{SO}_{4}$ (60 meses)

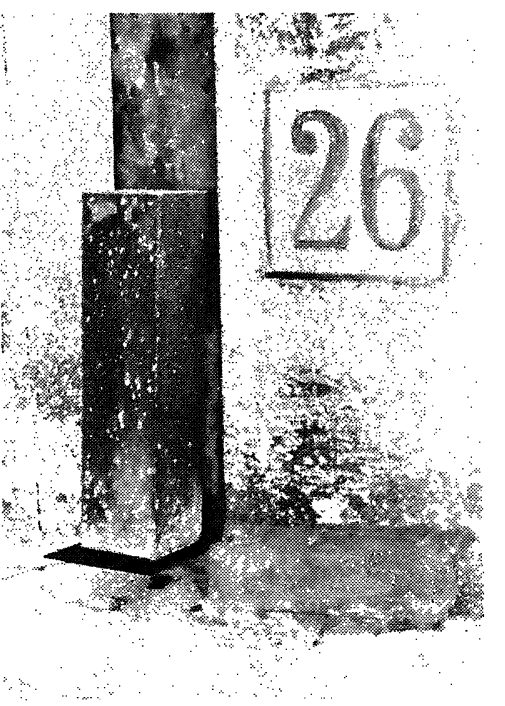

agua de mar (54 meses)

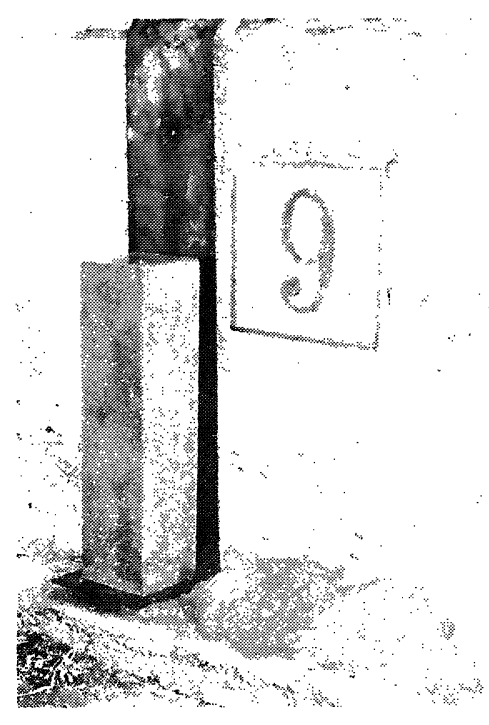

$\mathrm{MgSO}_{4} \cdot 7 \mathrm{H}_{2} \mathrm{O}$

(60 meses)

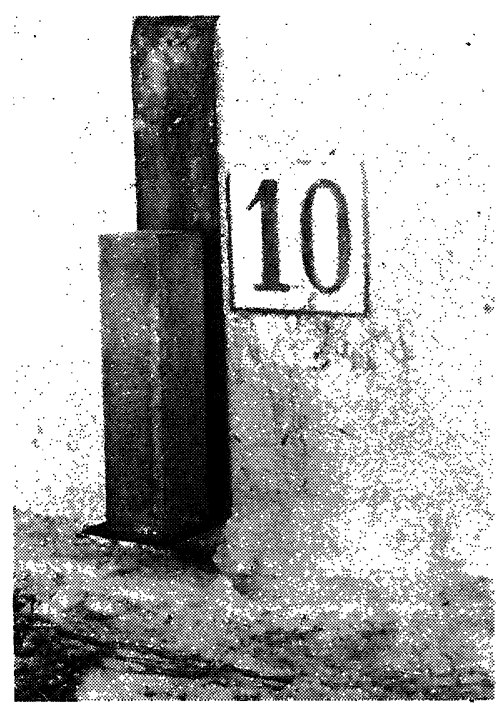

agua potable (60 meses)

Fig. 7.- Vista de las probetas de mortero de $7 \times 7 \times 28 \mathrm{~cm}$.

En la figura 9 se observa que las resistencias mecánicas a compresión de las probetas de hormigón sumergidas en las diversas disoluciones agresivas y en agua potable siguen una evolución parecida a lo largo de los cinco años, formando un haz en donde se entrecruzan dichos valores.

La disolución saturada de sulfato de calcio se ha renovado a los 8,14 y 22 meses por ser la concentración de iones sulfato inferior a $500 \mathrm{mg} / \mathrm{l}$.

El aspecto de las probetas sometidas a la acción del agua potable y de las disoluciones agresivas 


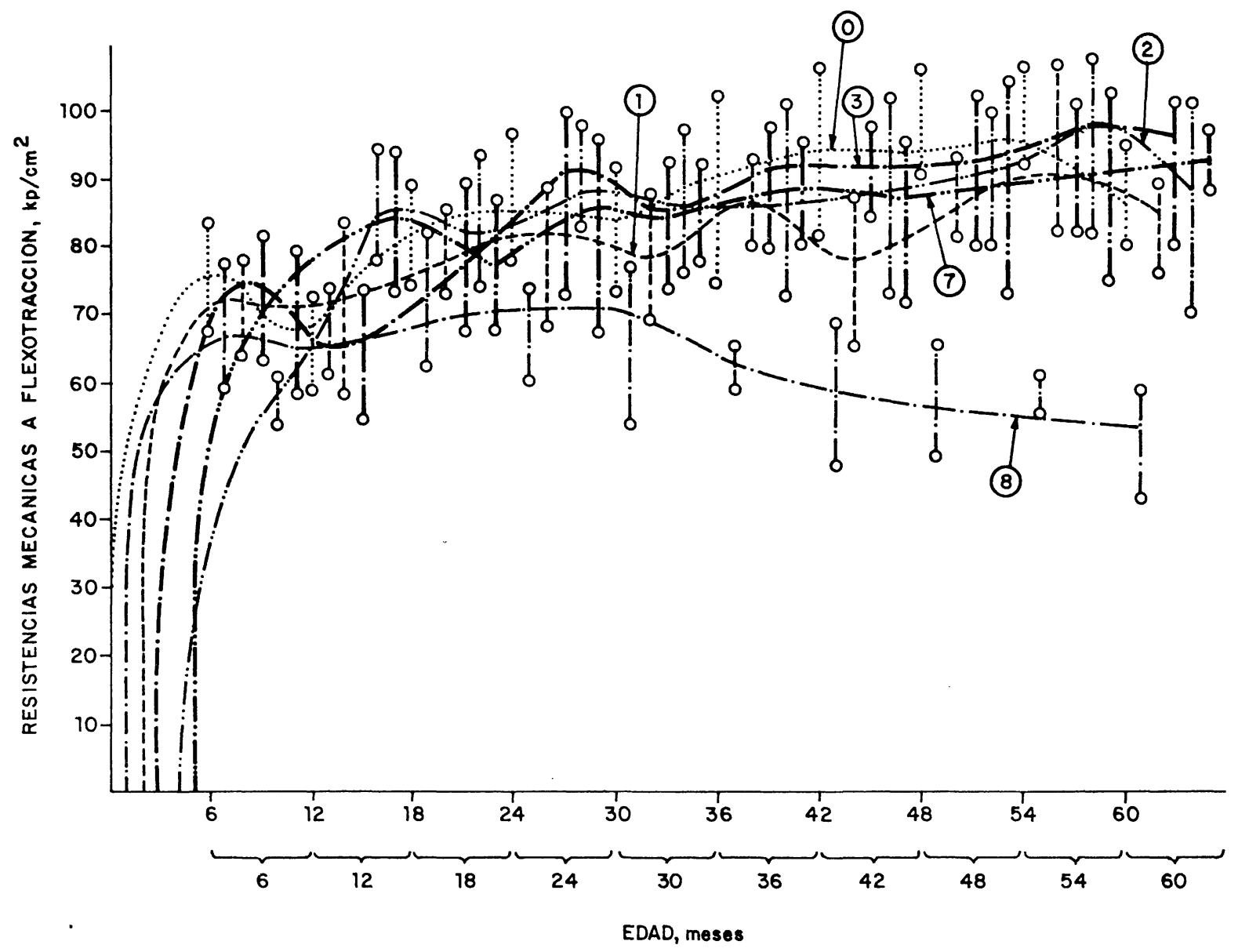

Fig. 8.-Evolución de las resistencias mecánicas, a flexotracción, de las probetas de hormigón de $10 \times 10 \times 40 \mathrm{~cm}$, sumergidas en:

1. disolución saturada de $\mathrm{CaSO}_{4} .2 \mathrm{H}_{2} \mathrm{O}$,

2. disolución de $\mathrm{Na}_{2} \mathrm{SO}_{4}(2,1 \mathrm{~g} / l)$,

3. disolución de $\mathrm{MgSO}_{4} .7 \mathrm{H}_{2} \mathrm{O}(3,22 \mathrm{~g} / l)$,

7. disolución de $\mathrm{Na}_{2} \mathrm{SO}_{4}(3,5 \mathrm{~g} / l)+\mathrm{NaCl}(20,0 \mathrm{~g} / l)$,

8. agua de mar artificial ASTM D 1141-75.

0 . agua potable.

era normal a todas las edades como se puede apreciar en la figura 10 en donde, a titulo de ejemplo, se encuentra una fotografia de una de las probetas de las distintas series sometidas durante 5 años a la acción del agua potable y de las mencionadas disoluciones agresivas. Las probetas sometidas a la acción del agua de mar aparecen recubiertas de una capa de $\operatorname{Mg}(\mathrm{OH})_{2}$.

Por otra parte, se ha hecho el estudio por difracción de rayos $\mathrm{X}$ de la fracción enriquecida (pasta de cemento) extraída de cada uno de los dos trozos de las probetas de hormigón (superior e inferior), sumergidas durante diversos periodos de tiempo en agua potable y en las disoluciones agresivas; dichas probetas corresponden a las que han dado las mayores resistencias mecánicas a flexotracción.

En la figura 11 se encuentran los diagramas de difracción de rayos $\mathrm{X}$, correspondientes a los dos trozos mencionados de las series de probetas sumergidas durante 360 dias y en la tabla 2 la intensidad de los picos (cuentas) de los compuestos cristalinos ettringita, portlandita y calcita, para $2 \theta=8,9^{\circ}-18,0^{\circ}$ y $29,5^{\circ}$, respectivamente; intensidad que se ha referido a la del pico del $\alpha-\mathrm{SiO}_{2}$, que aparece a $2 \theta=26,7^{\circ}$, en el DRX de la muestra extraida de uno de los trozos de la probeta sumergida en agua potable filtrada durante 360 dias. 


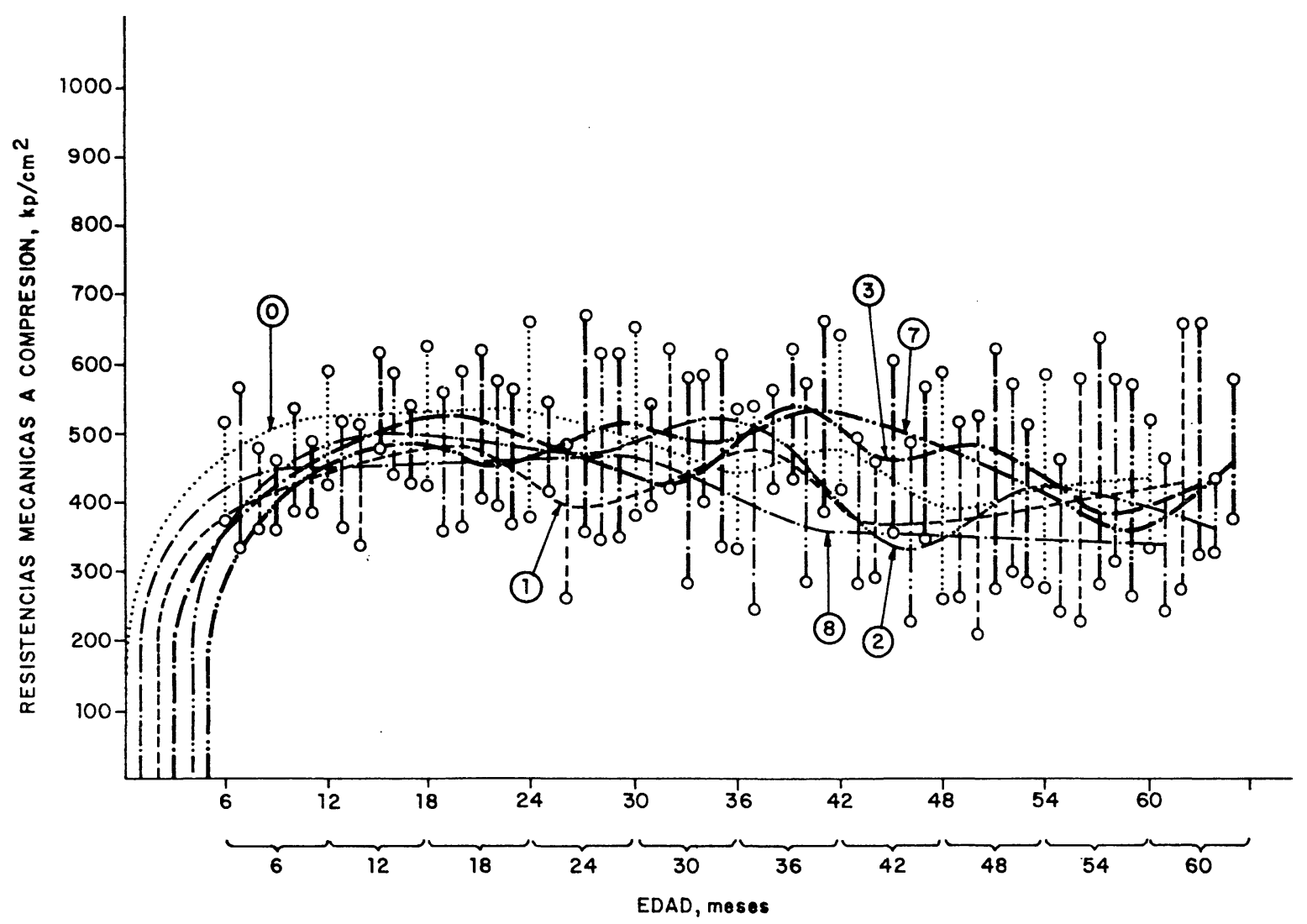

Fig. 9.-Evolución de las resistencias mecánicas, a compresión, de las probetas de hormigón de $10 \times 10 \times 40 \mathrm{~cm}$, sumergidas en:

1. disolución saturada de $\mathrm{CaSO}_{4} \cdot 2 \mathrm{H}_{2} \mathrm{O}$,

2. disolución de $\mathrm{Na}_{2} \mathrm{SO}_{4}(2,1 \mathrm{~g} / l)$,

3. disolución de $\mathrm{MgSO}_{4} .7 \mathrm{H}_{2} \mathrm{O}(3,22 \mathrm{~g} / l)$,

7. disolución de $\mathrm{Na}_{2} \mathrm{SO}_{4}(3,5 \mathrm{~g} / l)+\mathrm{NaCl}(20,0 \mathrm{~g} / l)$,

8. agua de mar artificial ASTM D 1141-75,

0 . agua potable.

En los diagramas de difracción de rayos $\mathrm{X}$ anteriores (fig. 11) y en la tabla de intensidades (tabla 2) se observa:

TABLA 2

$D R X$; intensidades relativas de los picos de ettringita $\left(2 \theta=8,9^{\circ}\right)$, portlandita $\left(2 \theta=18,0^{\circ}\right)$ $y$ calcita $\left(2 \theta=29,5^{\circ}\right)$

\begin{tabular}{|c|c|c|c|c|c|c|c|c|c|c|c|c|}
\hline \multirow[t]{2}{*}{ Compuesto } & \multicolumn{2}{|c|}{$\begin{array}{c}\text { Agua } \\
\text { potable }\end{array}$} & \multicolumn{2}{|c|}{$\begin{array}{c}\begin{array}{c}\text { Disolución } \\
\text { saturada } \\
\text { de }\end{array} \\
\mathrm{CaSO}_{4} \cdot 2 \mathrm{H}_{2} \mathrm{O}\end{array}$} & \multicolumn{2}{|c|}{$\begin{array}{c}\text { Disolución } \\
\text { de } \\
\mathrm{Na}_{2} \mathrm{SO}_{4}\end{array}$} & \multicolumn{2}{|c|}{$\begin{array}{c}\begin{array}{c}\text { Disolución } \\
\text { de }\end{array} \\
\mathrm{MgSO}_{4} .7 \mathrm{H}_{2} \mathrm{O}\end{array}$} & \multicolumn{2}{|c|}{$\begin{array}{c}\text { Disolución } \\
\text { de } \mathrm{Na}_{2} \mathrm{SO}_{4} \\
+ \\
\mathrm{NaCl}\end{array}$} & \multicolumn{2}{|c|}{$\begin{array}{c}\text { Agua de } \\
\text { mar artificial } \\
\text { ASTM } \\
\text { D 1141-7 }\end{array}$} \\
\hline & Inferior & Superior & Inferior & Superior & Inferior & Superior & Inferior & Superior & Inferior & Superior & Inferior & Superior \\
\hline Ettringita & 217 & 243 & 223 & 242 & 194 & 173 & 223 & 231 & 219 & 194 & 215 & 304 \\
\hline Portlandita & 1.080 & 918 & 1.403 & 698 & 682 & 549 & 1.242 & 969 & 818 & 396 & 1.056 & 693 \\
\hline Calcita & 519 & 462 & 517 & 433 & 545 & 508 & 339 & 647 & 957 & 731 & 159 & 427 \\
\hline
\end{tabular}






$\mathrm{CaSO}_{4} \cdot 2 \mathrm{H}_{2} \mathrm{O}$; sat.

(60 meses)

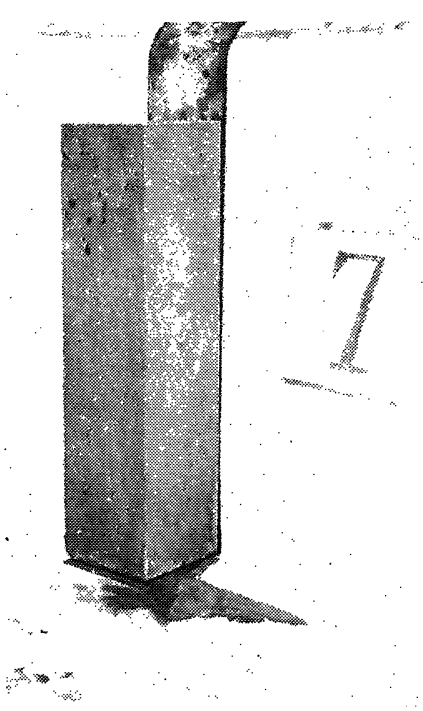

$\mathrm{Na}_{2} \mathrm{SO}_{4}+\mathrm{NaCl}$

(60 meses)

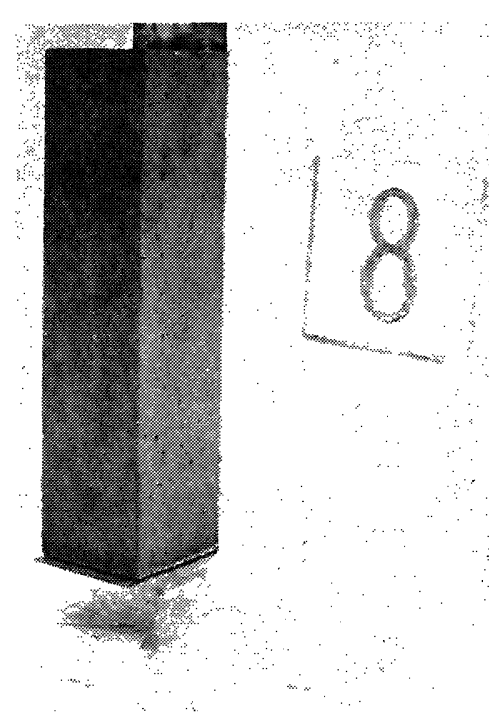

$\mathrm{Na}_{2} \mathrm{SO}_{4}$

(60 meses)

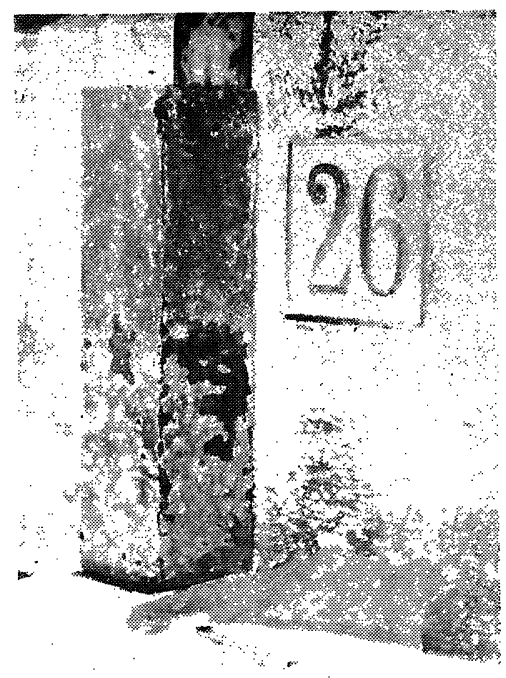

agua de mar

(54 meses)

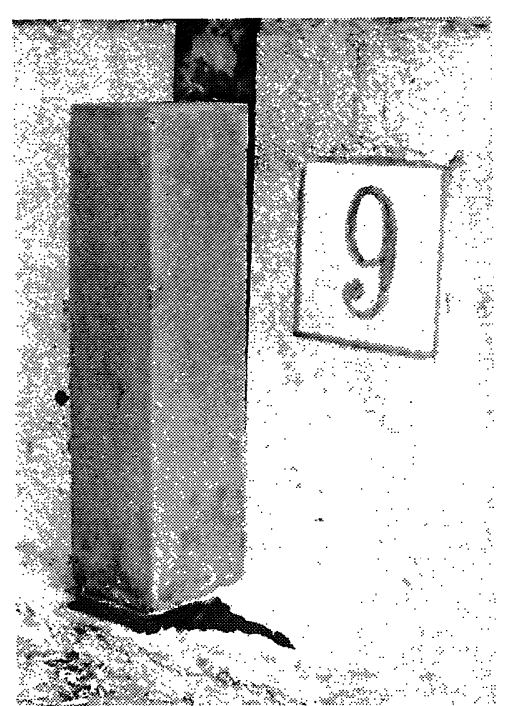

$\mathrm{MgSO}_{4} \cdot 7 \cdot \mathrm{H}_{2} \mathrm{O}$

(60 meses)

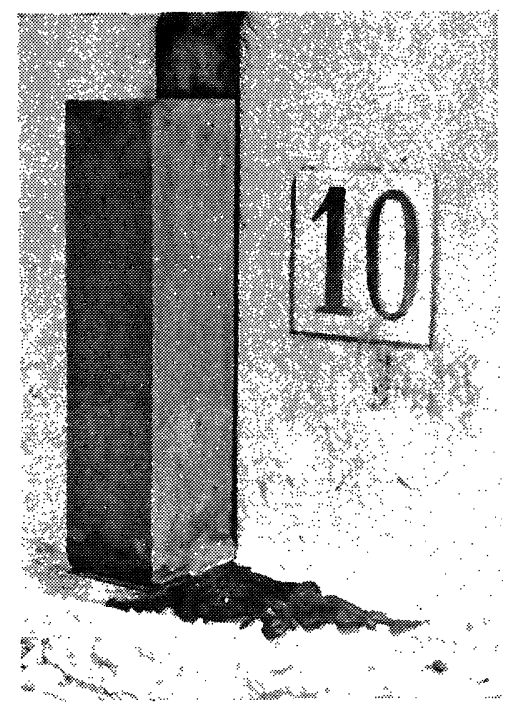

agua potable (60 meses)

Fig. 10.-Vista de las probetas de hormigón de $10 \times 10 \times 40 \mathrm{~cm}$.

1. En los diagramas de difracción de rayos $X$ de las distintas series de probetas

a) que la intensidad del pico de la ettringita es de la misma magnitud en todos los DRX, excepto en los diagramas de las zonas superiores de las probetas sumergidas en agua de mar ASTM (es superior) y en la disolución de $\mathrm{Na}_{2} \mathrm{SO}_{4}$ (es menor).

b) que la intensidad del pico de la portlandita, es aproximadamente la misma en los diagramas de las zonas inferiores, excepto para el caso de los DRX de las probetas sumergidas en las disoluciones de $\mathrm{Na}_{2} \mathrm{SO}_{4}+\mathrm{NaCl}$ y de $\mathrm{Na}_{2} \mathrm{SO}_{4}$. En los diagramas de la 

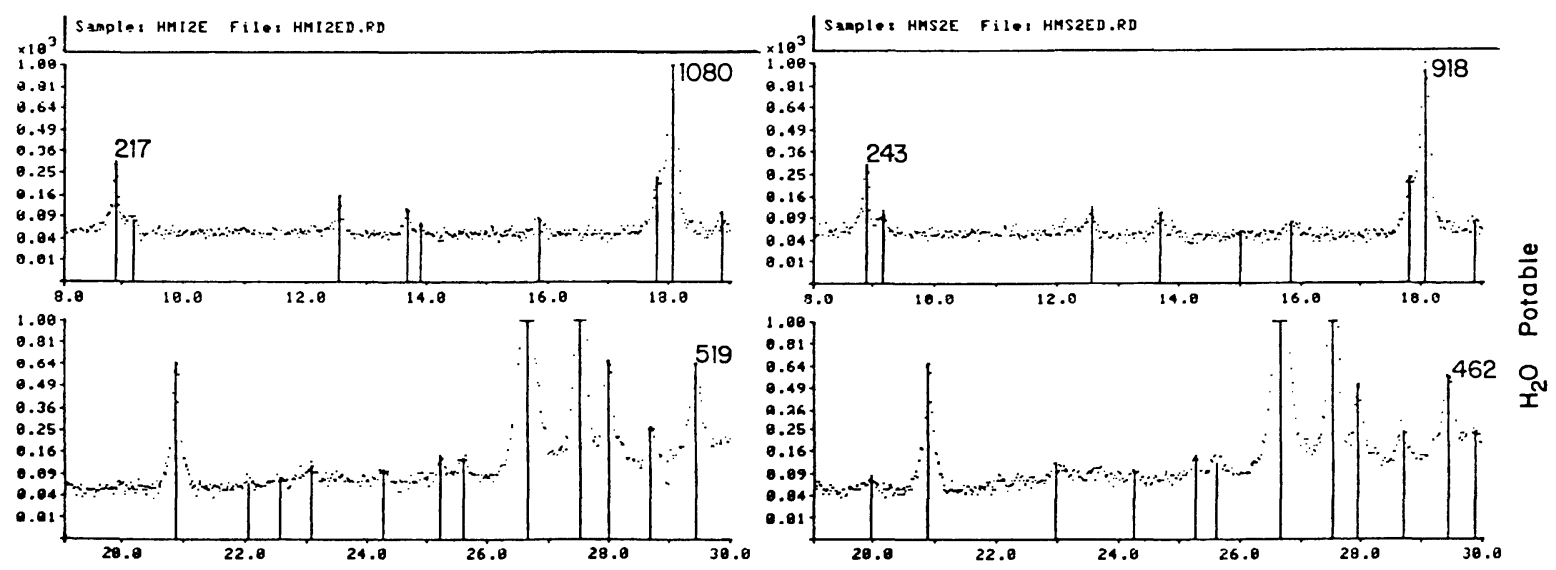

Hormigón máx. rotura: zona inferior.

Hormigón máx. rotura: zona superior.
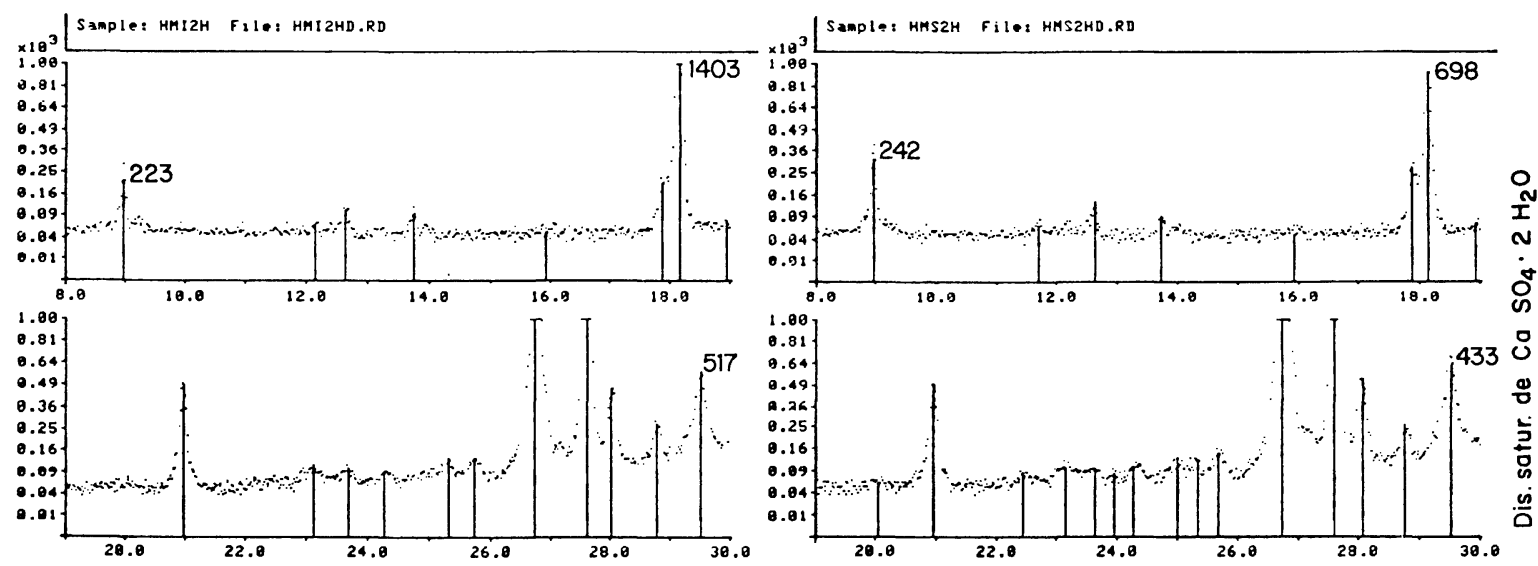

Hormigón máx. rotura: zona inferior.

Hormigón máx. rotura: zona superior.

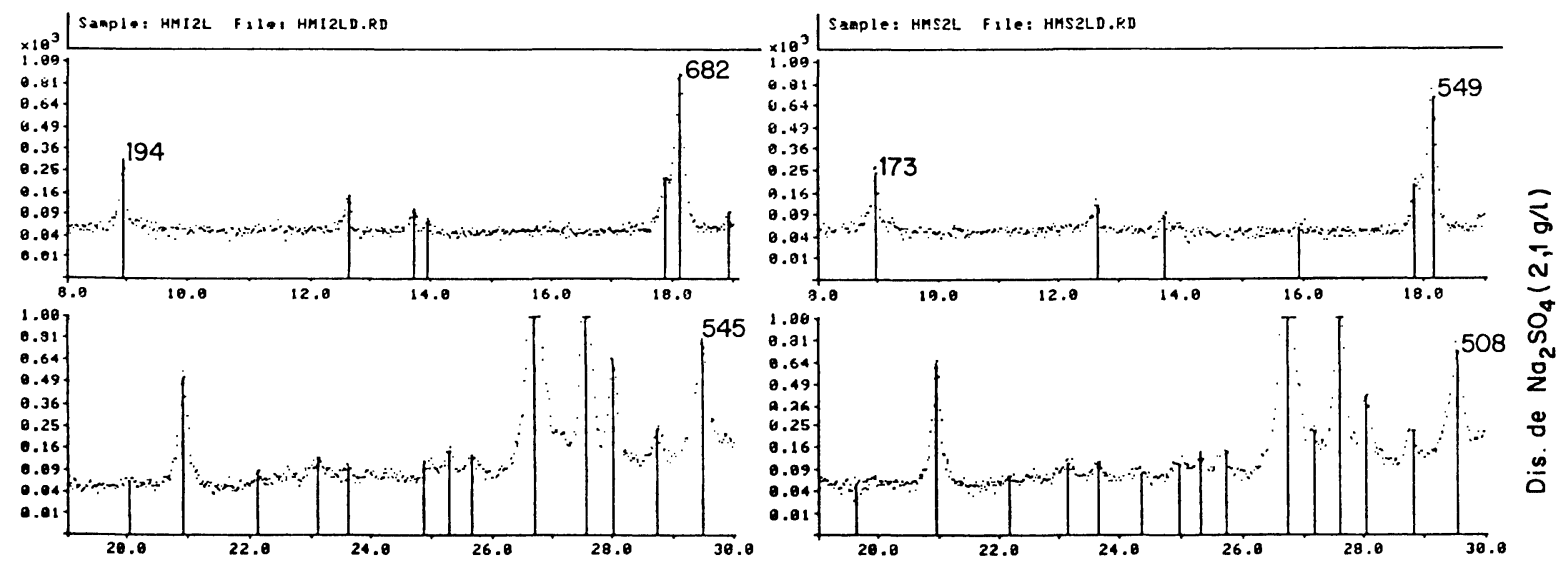

Fig. 11.-DRX de la fracción enriquecida (pasta de cemento) extraida de la zona inferior y de la zona superior de las probetas de hormigón sumergida en agua potable y en las disoluciones agresivas. 

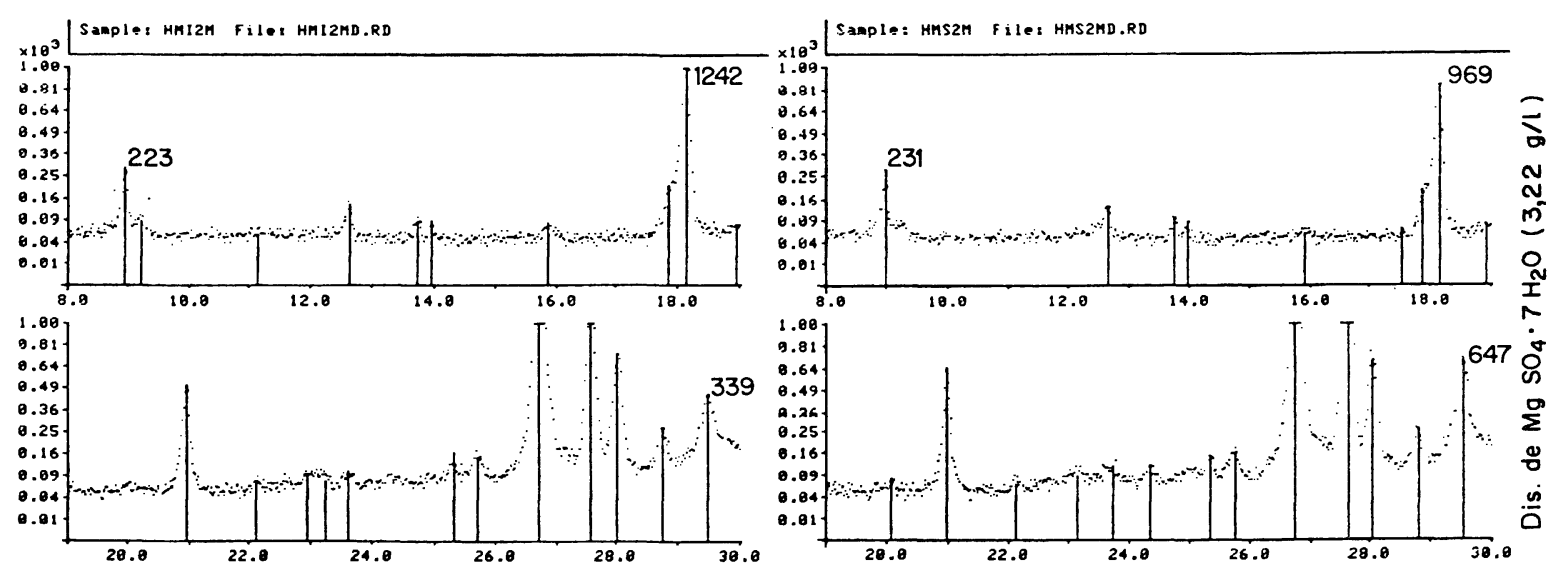

Hormigón máx. rotura: zona inferior.

Hormigón máx. rotura: zona superior.
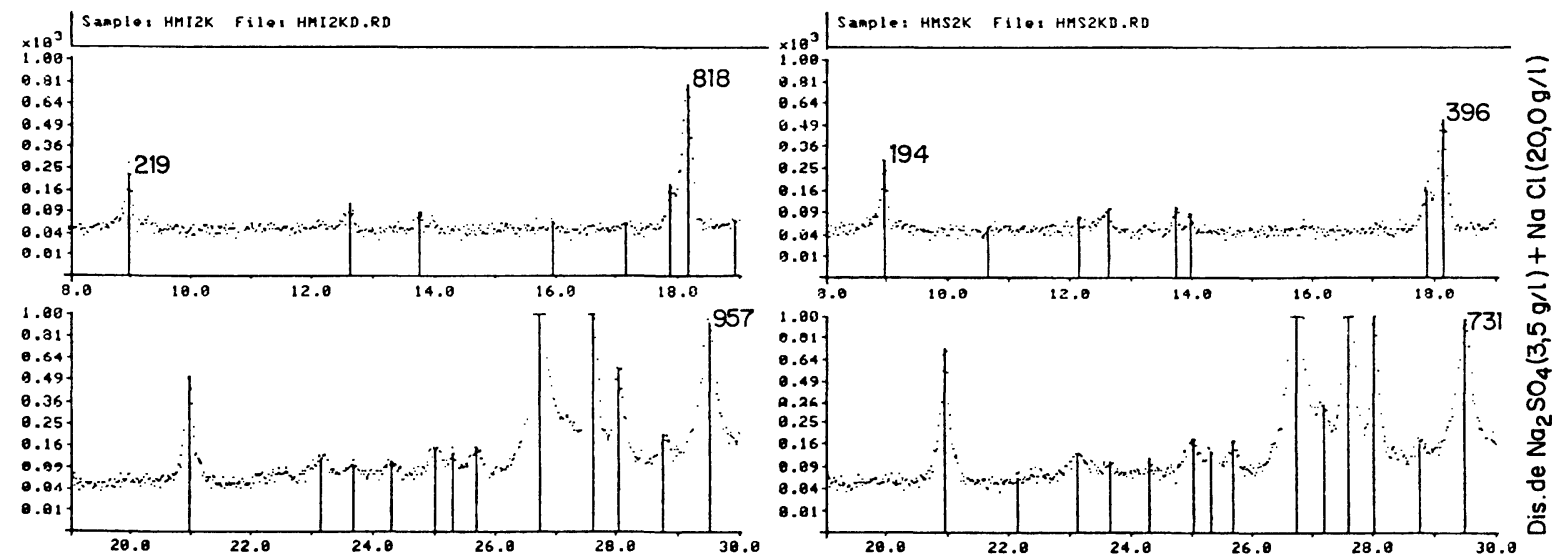

Hormigón máx. rotura: zona inferior.

Hormigón máx. rotura: zona superior.
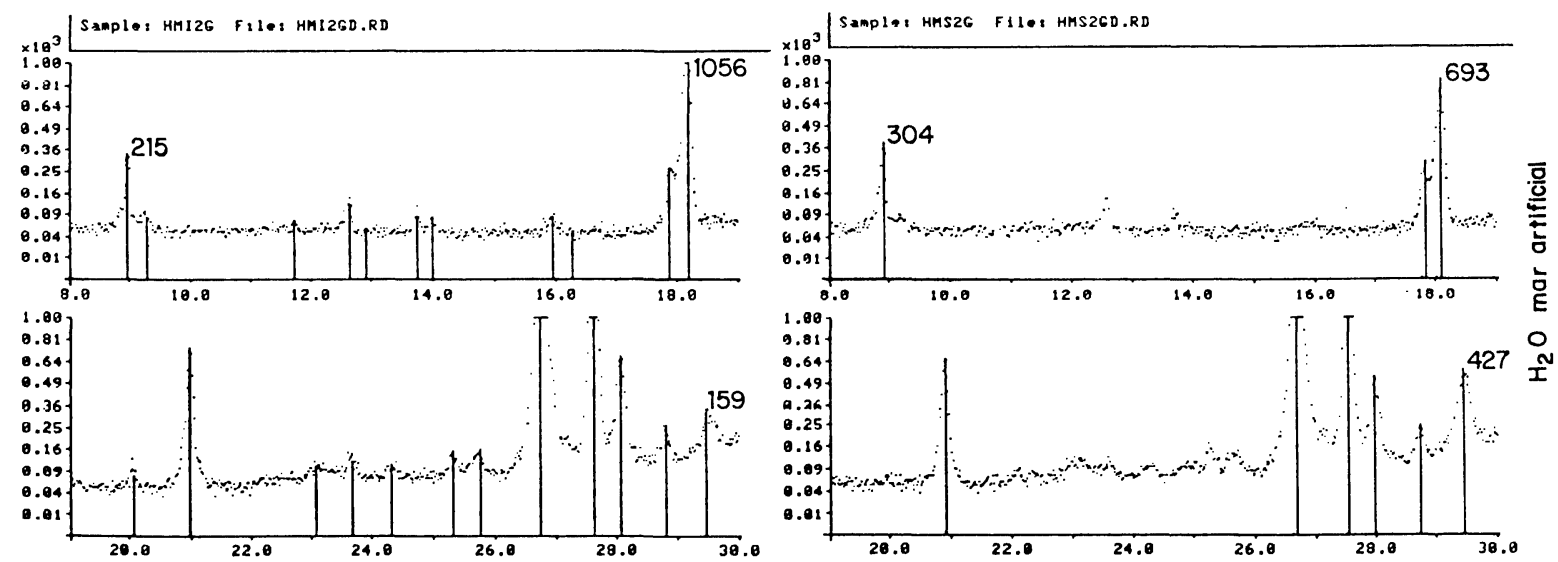

Fig. 11.-DRX de la fracción enriquecida (pasta de cemento) extraida de la zona inferior y de la zona superior de las probetas de hormigón sumergida en agua potable y en las disoluciones agresivas. 
zona superior es distinta, dicha intensidad, presentando la siguiente graduación, de acuerdo con los medios en donde han estado sumergidas las probetas: disolución de $\mathrm{MgSO}_{4} \cdot 7 \mathrm{H}_{2} \mathrm{O}>$ $>$ Agua $>$ disolución saturada de $\mathrm{CaSO}_{4} \cdot 2 \mathrm{H}_{2} \mathrm{O} \simeq$ agua de mar ASTM $>$ disolución de $\mathrm{Na}_{2} \mathrm{SO}_{4}>$ disolución de $\mathrm{Na}_{2} \mathrm{SO}_{4}+\mathrm{NaCl}$,

c) que la intensidad del pico de la calcita en los diagramas de difracción de rayos $\mathrm{X}$ mencionados es distinta, según la disolución en donde han estado sumergidas las distintas series de probetas; asi, en el caso de los diagramas de la zona superior, se puede establecer la siguiente graduación, según el medio en donde se han conservado las probetas: disolución de $\mathrm{Na}_{2} \mathrm{SO}_{4}+\mathrm{NaCl}>$ disolución de $\mathrm{MgSO}_{4} .7 \mathrm{H}_{2} \mathrm{O}>$ disolución de $\mathrm{Na}_{2} \mathrm{SO}_{4}>$ agua potable $\simeq$ disolución saturada de $\mathrm{CaSO}_{4} \cdot 2 \mathrm{H}_{2} \mathrm{O} \simeq$ agua de mar ASTM y en el caso de los diagramas de la zona inferior: disolución de $\mathrm{Na}_{2} \mathrm{SO}_{4}+\mathrm{NaCl}>$ disolución de $\mathrm{Na}_{2} \mathrm{SO}_{4} \simeq$ agua potable $\simeq$ disolución saturada de $\mathrm{CaSO}_{4} .2 \mathrm{H}_{2} \mathrm{O}>$ disolución de $\mathrm{MgSO}_{4} .7 \mathrm{H}_{2} \mathrm{O}>$ agua de mar ASTM.

2. En los diagramas de difracción de rayos $X$ de la misma probeta

a) que la intensidad del pico de la ettringita es de magnitud análoga en los DRX de las dos zonas de una probeta, excepto en los diagramas de las probetas sumergidas en agua de mar ASTM (I, zona superior > I, zona inferior),

b) que la intensidad del pico de la portlandita en los DRX de la zona inferior es mayor que la correspondiente a los diagramas de la zona superior,

c) que la intensidad del pico de la calcita de los DRX de las muestras extraidas de la zona inferior de las probetas es menor que la de los diagramas de la zona superior de las probetas sumergidas en la disolución de $\mathrm{MgSO}_{4} .7 \mathrm{H}_{2} \mathrm{O}$ y en agua de mar artificial ASTM; en los restantes casos dicha intensidad es mayor.

\section{CONCLUSIONES}

\section{Primera}

La evolución de las resistencias mecánicas a flexotracción de las distintas series de probetas de mortero de $1 \times 1 \times 6 \mathrm{~cm}$, de $4 \times 4 \times 16 \mathrm{~cm}$ y de $7 \times 7 \times 28 \mathrm{~cm}$ y de hormigón de $10 \times 10 \times 40 \mathrm{~cm}$, hechas con el cemento portland resistente a los sulfatos (objeto de este trabajo) sumergidas, después del periodo de curado, en agua potable, en agua potable filtrada y en las diversas disoluciones agresivas estudiadas hasta 3 años las probetas de mortero de $1 \times 1 \times 6 \mathrm{~cm}$ y hasta 5 años las distintas series de probetas restantes, es función de la composición química de dichas disoluciones.

\section{Segunda}

Las resistencias mecánicas, a flexotracción, de las series de probetas de mortero de $1 \times 1 \times 6 \mathrm{~cm}$ sumergidas en la disolución saturada de $\mathrm{CaSO}_{4} \cdot 2 \mathrm{H}_{2} \mathrm{O}$, en la disolución saturada de $\mathrm{CaSO}_{4} \cdot 2 \mathrm{H}_{2} \mathrm{O}+$ $+\mathrm{MgSO}_{4} \cdot 7 \mathrm{H}_{2} \mathrm{O}(32,2 \mathrm{~g} / l)$ y en la disolución de $\mathrm{MgSO}_{4} .7 \mathrm{H}_{2} \mathrm{O}(32,2 \mathrm{~g} / \mathrm{l})$ son superiores, a todas las edades, que las correspondientes a las de las probetas conservadas bajo agua potable filtrada y éstas del mismo orden que las de las series sumergidas en la disolución saturada de $\mathrm{CaSO}_{4} \cdot 2 \mathrm{H}_{2} \mathrm{O}+\mathrm{MgSO}_{4} \cdot 7 \mathrm{H}_{2} \mathrm{O}(3,22 \mathrm{~g} / l)$ y en la disolución de $\mathrm{MgSO}_{4} \cdot 7 \mathrm{H}_{2} \mathrm{O}(3,22 \mathrm{~g} / l)$.

Las resistencias mecánicas, a flexotracción, de las series de probetas sumergidas en agua de mar artificial son, a partir de los 24 meses, del mismo orden que las de las series sometidas a la 
acción de las disoluciones de $\mathrm{MgSO}_{4} .7 \mathrm{H}_{2} \mathrm{O}(3,22 \mathrm{~g} / \mathrm{l})$ y saturada de $\mathrm{CaSO}_{4} \cdot 2 \mathrm{H}_{2} \mathrm{O}+$ $+\mathrm{MgSO}_{4} .7 \mathrm{H}_{2} \mathrm{O}(3,22 \mathrm{~g} / l)$.

\section{Tercera}

Las resistencias mecánicas a flexotracción de las series de probetas de mortero de $4 \times 4 \times 16 \mathrm{~cm}$ sumergidas en las diversas disoluciones, excepto en agua de mar artificial, son mayores que las de las probetas conservadas en agua potable.

Las resistencias mecánicas a compresión de las probetas sumergidas en las disoluciones de sulfato de calcio (saturada), sulfato de sodio $(2,1 \mathrm{~g} / l)$ y sulfato de magnesio $(3,22 \mathrm{~g} / l)$ son superiores que las de las series conservadas en agua potable, menores - por regla general- las conservadas en la disolución de $\mathrm{Na}_{2} \mathrm{SO}_{4}(3,5 \mathrm{~g} / l)+\mathrm{NaCl}(20,0 \mathrm{~g} / l)$ hasta 48 meses y ligeramente superiores a partir de esta edad.

Las resistencias de las distintas series de probetas sometidas a la acción del agua de mar artificial son menores.

\section{Cuarta}

Las resistencias mecánicas a flexotracción de las series de probetas de hormigón de $10 \times 10 \times 40$ $\mathrm{cm}$ sumergidas en la disolución de $\mathrm{MgSO}_{4} .7 \mathrm{H}_{2} \mathrm{O}(3,22 \mathrm{~g} / l)$ son ligeramente menores, o del mismo orden, que las correspondientes a las de las probetas conservadas en agua potable y éstas superiores que las de las series sumergidas en las restantes disoluciones.

Las resistencias mecánicas a compresión de las series de probetas sumergidas en las diversas disoluciones son menores que las de las probetas conservadas bajo agua potable, excepto para las probetas sumergidas en las disoluciones de $\mathrm{MgSO}_{4} .7 \mathrm{H}_{2} \mathrm{O}(3,22 \mathrm{~g} / l)$ y de $\mathrm{Na}_{2} \mathrm{SO}_{4}(3,5 \mathrm{~g} / l)+$ $+\mathrm{NaCl}(20,0 \mathrm{~g} / l)$, en el periodo de tiempo comprendido entre 30 y 54 meses, que son superiores.

\section{Quinta}

Las resistencias mecánicas a tracción centrifuga de las series de probetas de mortero de $7 \times 7 \times 28 \mathrm{~cm}$ sumergidas en las disoluciones de $\mathrm{Na}_{2} \mathrm{SO}_{4}(2,1 \mathrm{~g} / l)$ y de $\mathrm{MgSO}_{4} \cdot 7 \mathrm{H}_{2} \mathrm{O}$ $(3,22 \mathrm{~g} / l)$ son superiores que las correspondientes a las de las series de probetas conservadas en agua potable y éstas del mismo orden o ligeramente superiores que las de las series sumergidas en la disolución saturada de $\mathrm{CaSO}_{4} \cdot 2 \mathrm{H}_{2} \mathrm{O}$, en el primer caso, o en las disoluciones de $\mathrm{Na}_{2} \mathrm{SO}_{4}$ $(3,5 \mathrm{~g} / l)+\mathrm{NaCl}(20,0 \mathrm{~g} / l)$ y en agua de mar artificial en el segundo.

\section{BIBLIOGRAFIA}

(1) GASPAR-TEBAR, D.; SAGRERA-MORENO, J. L. y GONZALEZ-VILA, V. (1985): Contribución al estudio de la resistencia quimica de los cementos. Comportamiento de un cemento portland resistente al yeso (1. ${ }^{\text {" }}$ parte) Materiales de Construcción, abril-mayo-junio, 198, 31-41.

(2) GASPAR-TEBAR, D. y SAGRERA-MORENO, J. L. (1980): Resistencia quimica del hormigón: V. Acción del agua de mar: influencia de la adición de escoria a un cemento portland resistente al yeso. Evolución de las resistencias mecánicas a flexotracción y del coeficiente de corrosión. Materiales de Construcción, julio-agosto-septiembre, 179, 59-79.

(3) SAGRERA-MORENO, J. L. y GASPAR-TEBAR, D. (1982): Resistencia quimica del hormigón: XVII. Acción de una disolución saturada de yeso: influencia de la adición de escoria a un cemento portland resistente al yeso. Evolución de las resistencias mecánicas a flexotracción y de los coeficientes de corrosion. Materiales de Construcción, julioagosto-septiembre. 187, 41-54. 
(4) SAGRERA-MORENO, J. L. y GASPAR-TEBAR. D. (1981): Resistencia quimica del hormigon: X. Influencia de la adición de escoria a un cemento portland resistente al yeso. Estudio por DRX del sistema cemento 2/escoria-agua potable filtrada. Materiales de Construcción. abril-mayo-junio, 182, 11-22.

(5) GASPAR-TEBAR. D. y SAGRERA-MORENO, J. L. (1982): Resistencia quimica del hormigón: XVIII. Acción del agua de mar: influencia de la adición de escoria a un cemento portland resistente al yeso. Estudio por DRX. Materiales de Construccion. octubre-noviembre-diciembre, 188, 37-58.

(6) SAGRERA-MORENO, J. L. y GASPAR-TEBAR. D. (1984): Resistencia quimica del hormigon: XXIII. Influencia de la adición de escoria a un cemento portland resistente al yeso. Estudio de la concentración iónica del sistema cemento 2/escoria-agua potable filtrada. Materiales de Construcción, abril-mayo-junio, 194, 43-51.

(7) GASPAR-TEBAR. D. y SAGRERA-MORENO. J. L. (1984): Resistencia quimica del hormigon: XXIV. Influencia de la adición de escoria a un cemento portland resistente al yeso. Estudio de la concentración iónica del sistema cemento 2/escoria-agua de mar artificial. Materiales de Construcción. octubre-noviembre-diciembre. 196. 49-66.

(8) GASPAR-TEBAR. D. y SAGRERA-MORENO. J. L. (1977): Resistencia quimica del hormigon: I. Estudio de dos cementos portland frente al agua de mar. Materiales de Construcción. octubre-noviembre-diciembre. 168, 33-60.

\section{RE C O N O C I M I E N T O}

Nuestro más sincero agradecimiento a las personas del Equipo de Durabilidad del IETcc: Amalia Rodriguez Pereira, Lucila López Solana, Felipe Cantero Palacios y Manuel Cantero Palacios por su valiosa colaboración en la realización de este trabajo.

\section{publicaciones del i.e.t.c.c.}

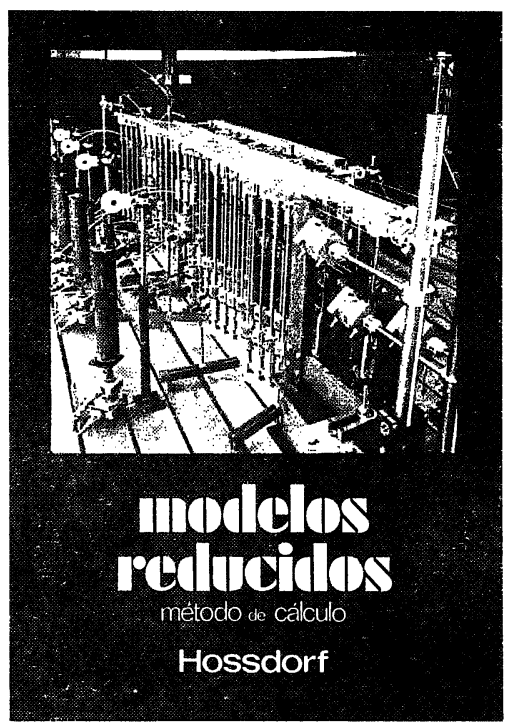

Modelos reducidos. Método de cálculo

H. Hossdorf, Ingeniero Civil

La técnica de los ensayos en modelos reducidos de estructuras sufre hoy dia una decisiva metamorfosis. Hasta hace poco era un medio más bien de artesania, que no siempre era tomado en serio por los académicos teorizantes Dara comprender el comportamiento resistente de las estructuras complejas y a que se acudio las más de las veces, como a un cias. Sin embargo, en poco tiempo y gracias a su cias. Sin embargo, en poco tiempo y gracias a su
conexion con los ordenadores digitales, se ha transformado en un instrumento cientificamente valioso, que no puede quedar a un lado en la práctica diaria del Ingeniero Proyectista.

Un volumen encuadernado en cartoné plastificado 250 páginas, 158 figuras y fotografias.

Precios: 1.800 ptas.; \$ USA 26.00

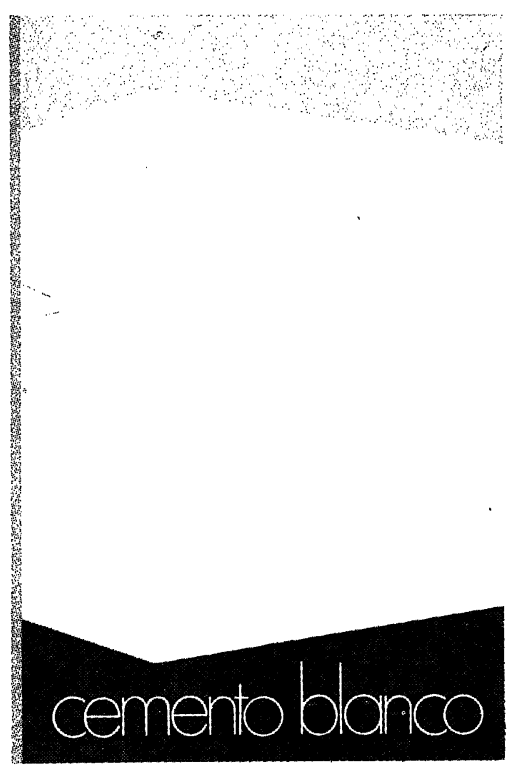

Cemento blanco

Julián Rezola

Ingeniero Quimico Dipl. I. Q. S.

Sabido es que existe una extensa y documentada bibliografia sobre el cemento gris: en cambio, no puede decirse lo mismo acerca del cemento portlan blanco, ya que los escritos existentes se refieren tan de aquél.

El autor nos ofrece sus profundos conocimientos y su larga experiencia tanto en laboratorio como en fabricación.

La parte descriptiva del libro se complementa con gráficos, diagramas y fotografias de gran utilidad, destinados a conseguir la aplicación apropiada de este aglomerante.

Un volumen encuadernado en cartoné policerado, de $17,4 \times 24,3 \mathrm{~cm}$. compuesto de 395 paginas. Precios: España, 1.700 ptas.: extranjero. $\$ 24.00$

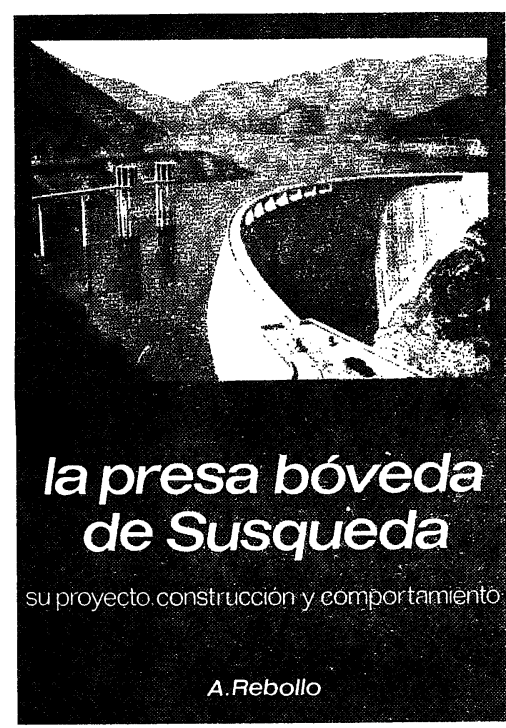

La presa bóveda de Susqueda

A. Rebollo,

Dr. Ingeniero de Caminos

El esfuerzo del constructor de presas se sitúa, por su pretension de perennidad, a contracorriente de las tendencias de la civilización actual, caracte. rizada por lo fungible. Pueden evocarse las 10.000 grandes presas en funcionamiento o en construcción que estan envejeciendo y reclaman los cuidados gerontologicos para mantener y perfeccionar su servicio y garantizar su inalienable pretensión de perennidad. En la medida en que todas nuevas obras. grandes o pequeñas, son portadoras de riesgos ecologicos $y$, a veces, calastrolicos, que aus presas cs todo un emplazo. La accion alogia de las presas es lodo un emplazo. La accion adelantada a Aruir para codos los que aman su propie obramion la devocion paternal que el ha puesto en Susqueda.

Un volumen encuadernado en cartone plastificado con lomo de tela, de $18 、 24,5 \mathrm{~cm}$. compuesto de 408 páginas, 330 figuras y fotografias y 39 tablas. Precios: 1.700 ptas.; extranjero, \$ USA 24.00 